Supporting Information for

\title{
Shape-Controlled Synthesis of Colloidal Nanorods and Nanoparticles of Barium Titanium Sulfide
}

\author{
Daniel Zilevu, Sidney E. Creutz* \\ Department of Chemistry, Mississippi State University, Mississippi State, MS 39762, United States \\ *screutz@chemistry.msstate.edu
}

\section{Table of Contents}

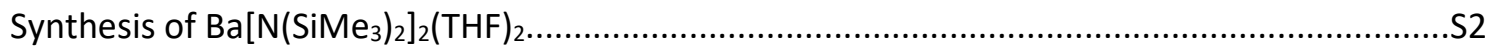

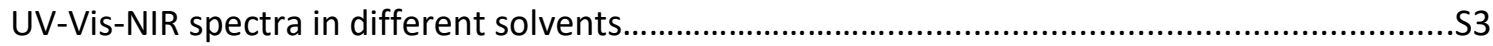

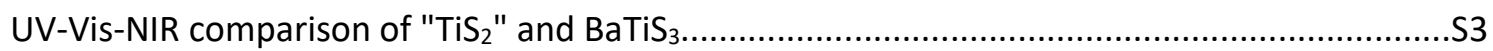

Additional XRD data: blank sample, phase comparison, impurities, orientation effects............S4

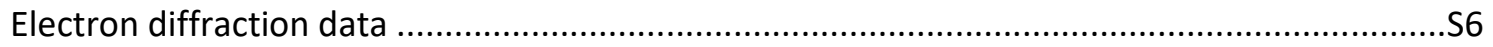

Table of reaction conditions for samples shown in Figure 12 (scatter plot).............................S6

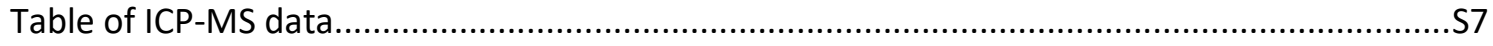

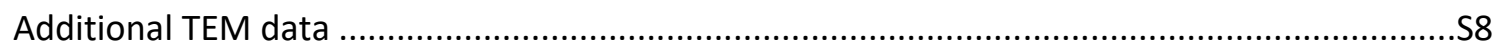

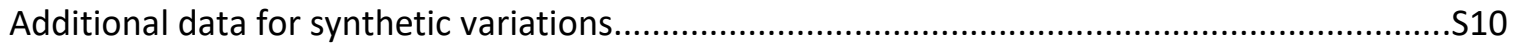

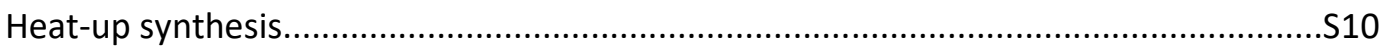

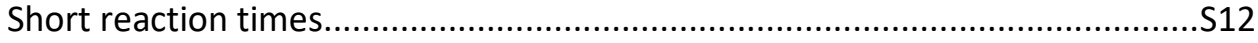

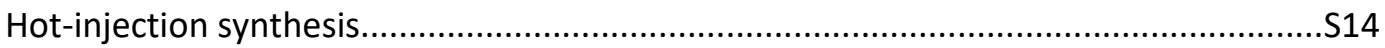

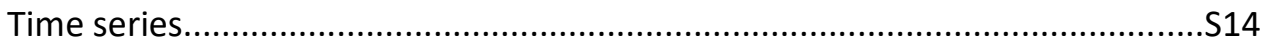

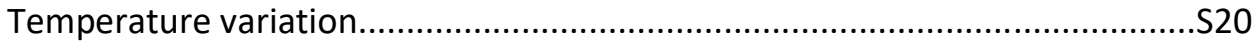

Concentration variation...................................................................... 26

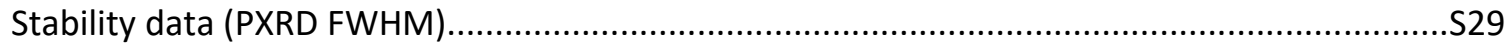

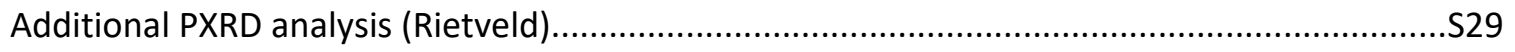

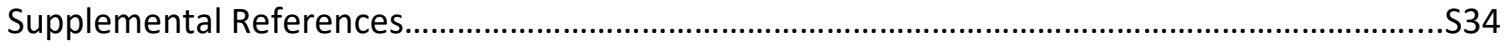




\section{Synthesis of $\mathrm{Ba}\left[\mathrm{N}\left(\mathrm{SiMe}_{3}\right)_{2}\right]_{2}(\mathrm{THF})_{2}$.}

The synthesis of the barium precursor $\mathrm{Ba}\left[\mathrm{N}\left(\mathrm{SiMe}_{3}\right)_{2}\right]_{2}(\mathrm{THF})_{2}$ follows that previously reported by Caulton et al. $^{1}$ In a typical reaction, $4.0 \mathrm{~g} \mathrm{(29} \mathrm{mmol)} \mathrm{of} \mathrm{barium} \mathrm{shavings} \mathrm{or} \mathrm{chunks} \mathrm{(cut} \mathrm{from} \mathrm{a} \mathrm{barium} \mathrm{rod)} \mathrm{are}$ added to a $100 \mathrm{~mL}$ pear-shaped Schlenk flask along with $25 \mathrm{~mL}$ of anhydrous THF and a Teflon stir bar under nitrogen or argon gas on a Schlenk line. To this mixture are added $24.0 \mathrm{~mL}$ (138 mmol, 4.8 eq.) of 1,1,1,3,3,3-hexamethyldisilazane $\left(\mathrm{HN}\left(\mathrm{SiMe}_{3}\right)_{2}\right.$ ) (standard reagent grade, purchased from Sigma Aldrich or Acros Organics and used without further purification). No immediate change is observed. Gaseous anhydrous ammonia is bubbled through the reaction mixture with vigorous stirring for approximately five minutes and then the reaction mixture is allowed to stir for approximately ten minutes undisturbed; this treatment is repeated four times. Bubbling ( $\mathrm{H}_{2}$ gas) is observed from the barium surface after each treatment. The reaction is then allowed to stir overnight ( 12 hours) under a static atmosphere of nitrogen gas. In the morning, if any solid barium metal remains in the reaction flask, ammonia is bubbled through the reaction mixture two additional times, followed by stirring for an additional five hours. During the course of the reaction, the barium metal pieces should be consumed and the reaction mixture becomes a suspension containing a gray/purple solid. The reaction mixture is then concentrated to dryness in vacuo and the flask containing the dried purplish-gray residue is transferred into a nitrogen glovebox where the rest of the workup/purification is carried out. The residue is extracted with pentane $(3 \times 50 \mathrm{~mL})$, which is filtered through Celite. The filtrate is concentrated to dryness again in vacuo, leaving a white solid. The white solid is taken up again in minimal pentane and stored in a freezer (-35 ${ }^{\circ} \mathrm{C}$ ) overnight, and the resulting microcrystalline solid, $\mathrm{Ba}\left[\mathrm{N}\left(\mathrm{SiMe}_{3}\right)_{2}\right]_{2}(\mathrm{THF})_{2}$, is isolated atop a groundglass frit. Typical yields are $40-60 \%$.

Note: Ammonia in this reaction is believed ${ }^{1}$ to act as a catalyst for the reaction of barium with the hexamethyldisilazane. Only brief bubbling of the ammonia through the solution is required, and should be repeated periodically until the barium metal is consumed. The gray-purple solid that forms during the course of the reaction was hypothesized ${ }^{1}$ to be insoluble $\mathrm{Ba}\left(\mathrm{NH}_{2}\right)_{2}$. Continuing ammonia bubbling for longer than necessary seems to result in increased formation of this byproduct, so careful monitoring of the barium metal consumption is required to maximize yields. However, this insoluble byproduct is readily removed by the filtration step during workup.

Caution! Barium metal reacts with water exothermically to produce $\mathrm{H}_{2}$ gas and presents a potential fire and explosion risk if not handled and disposed of properly. Ammonia gas is a toxic and highly irritating gas, and this reaction should be carried out with caution in a well-ventilated hood. 


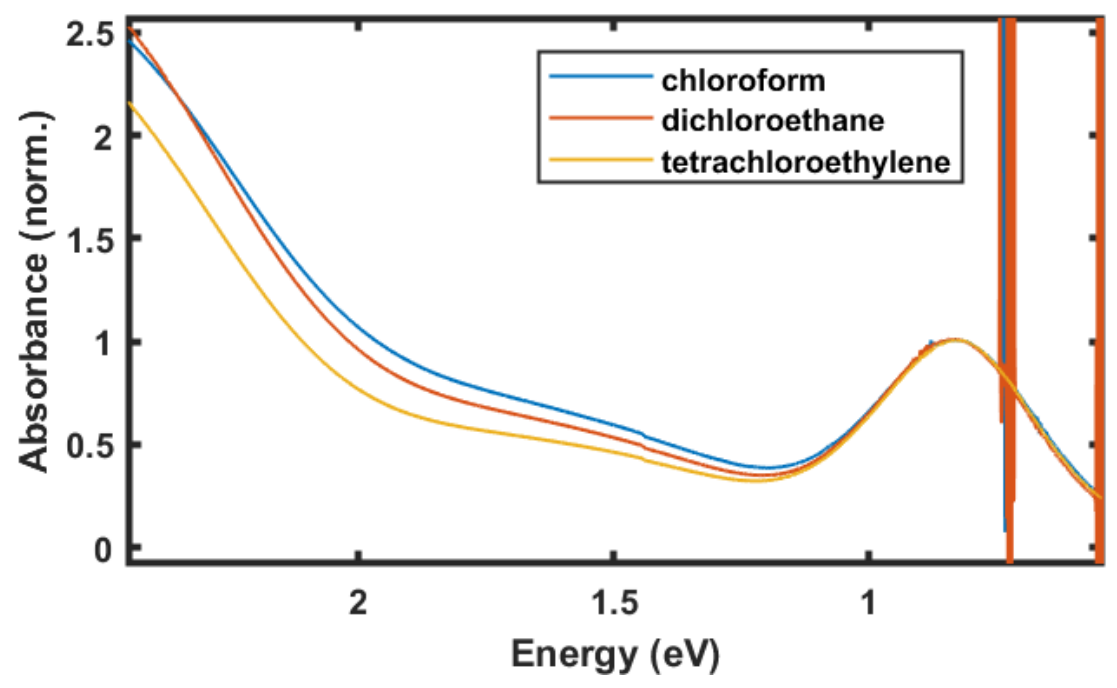

Figure S1. Solvent dependence of the absorbance of a sample of nanorods with width $=7 \mathrm{~nm}$. Absorbance spectra intensities are normalized to the max of the peak at $0.8 \mathrm{eV}$. There is no apparent dependence of the peak position on solvent for dichloroethane $\left(\varepsilon=10.36, \eta_{D}^{20}=1.4448\right)$, chloroform $(\varepsilon=$ $\left.4.81, \eta_{D}^{20}=1.4458\right)$, and tetrachloroethylene $\left(\varepsilon=2.5, \eta_{D}^{20}=1.5055\right)$.

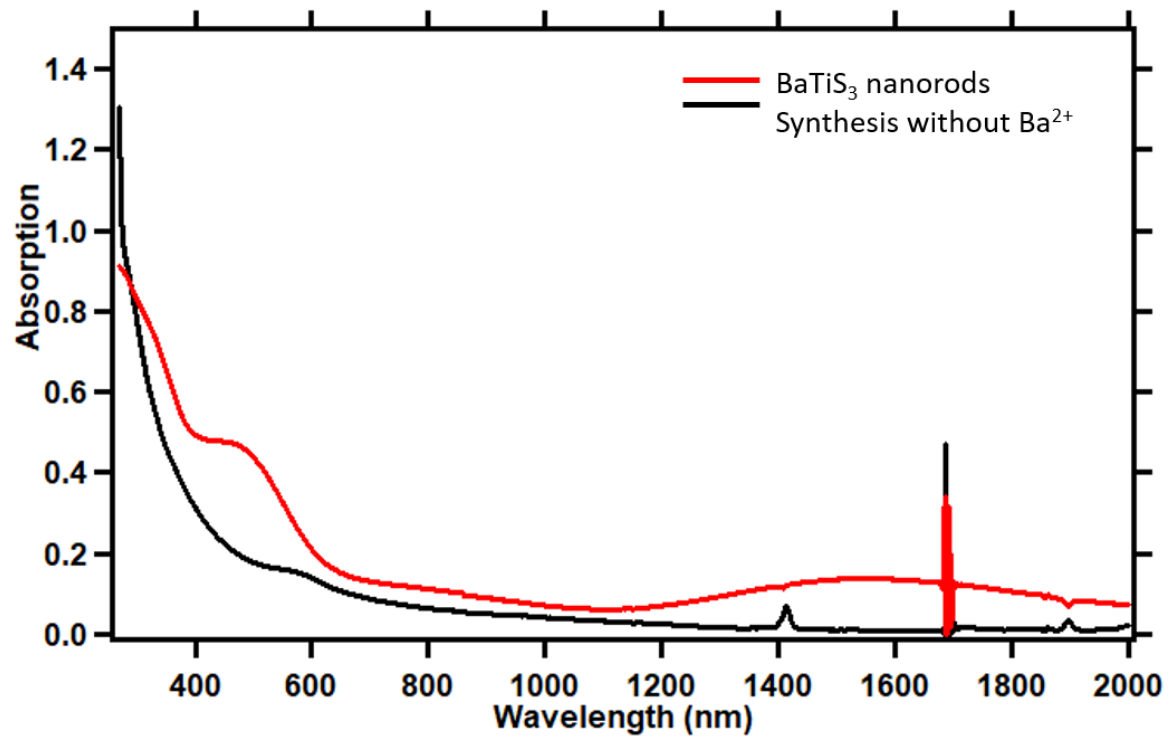

Figure S2. Comparison of the absorbance spectra of a representative sample of $\mathrm{BaTiS}_{3}$ nanorods with the material resulting from a synthesis run without including the barium precursor, $\mathrm{Ba}\left(\mathrm{N}(\mathrm{TMS})_{2}\right)_{2}(\mathrm{THF})_{2}$. The absorbance feature at $c a .580 \mathrm{~nm}$ from this synthesis is consistent with the spectroscopic properties reported for $\mathrm{TiS}_{2}$ nanoplatelets. ${ }^{2,3}$ The material from this reaction did not show clear diffraction features by PXRD and was not characterized further. 


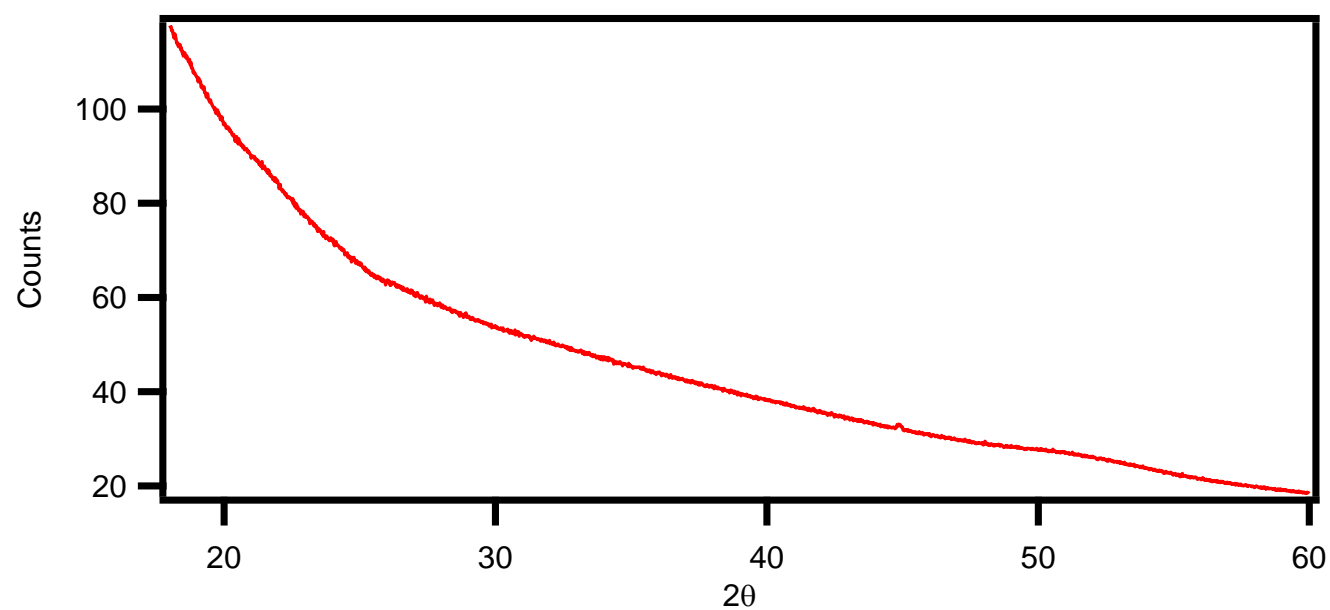

Figure S3. Powder XRD blank from "zero-background" silicon plate and sample holder. For data shown in the text, the sloping background was removed using a polynomial background correction.

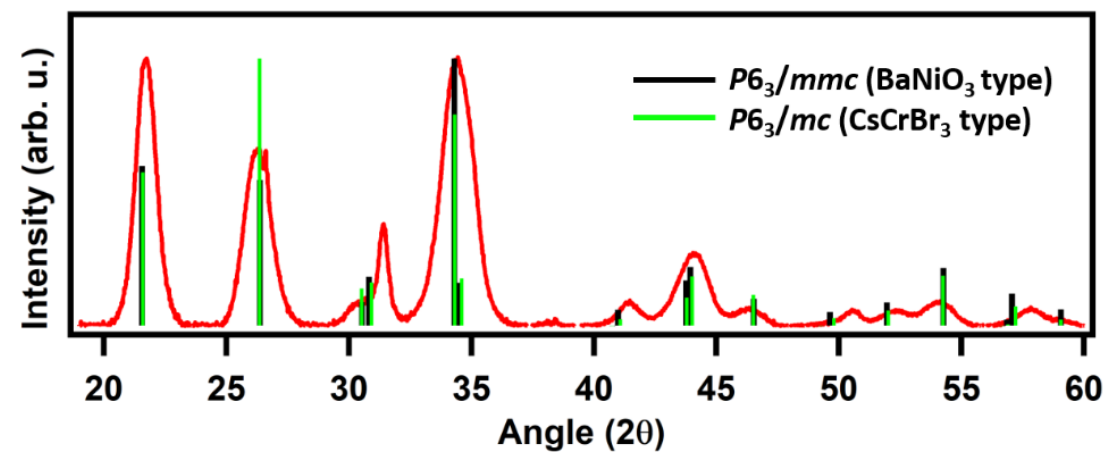

Figure S4. Overlay of the powder XRD data from $\mathrm{BaTiS}_{3}$ nanorods with the predict PXRD patterns for the $\mathrm{BaNiO}_{3}$ and $\mathrm{CsCrBr}_{3}$ structure types. ${ }^{4,5}$ The two structures are indistinguishable with respect to the data. 
Occasionally, additional features in the powder XRD are observed that may originate from impurities, such as the spurious peak observed in the figure below at $\sim 26.6^{\circ}$ (marked with a ${ }^{*}$ ). The identity of this possible impurity is unknown. However, this is not the norm and in most samples no peaks beyond those attributed to $\mathrm{BaTiS}_{3}$ or to the substrate background can be resolved in the PXRD data.

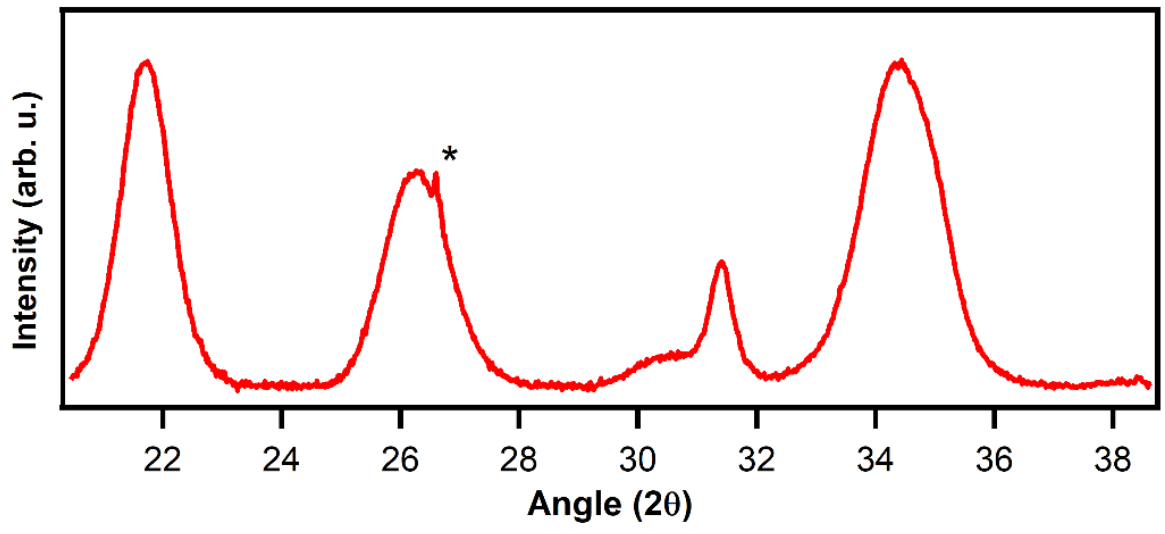

Figure S5. Example of PXRD data with an unknown impurity peak.
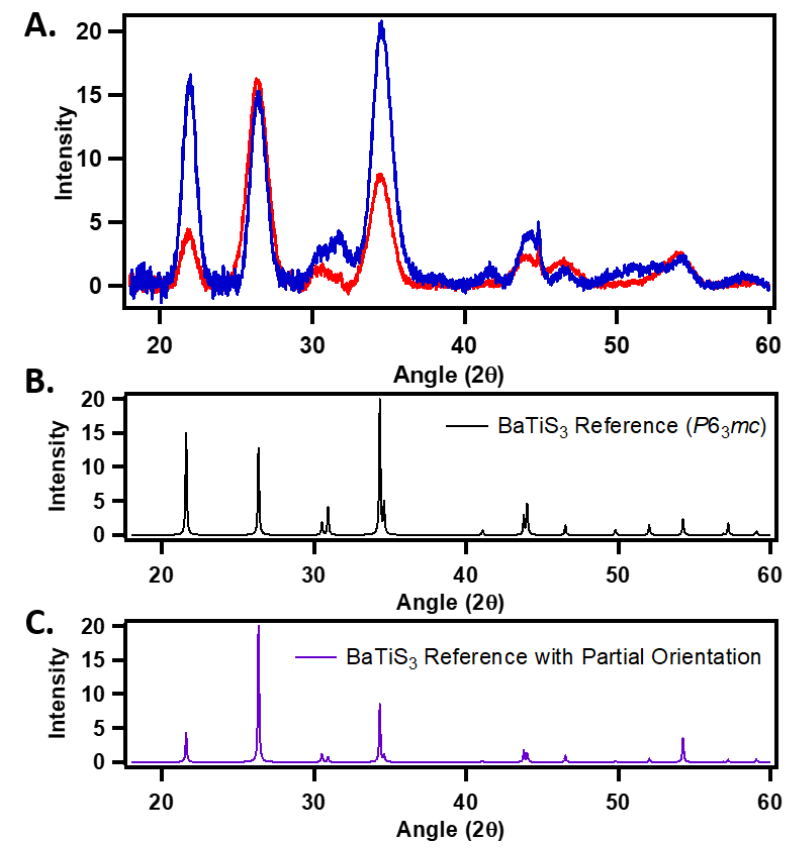

Figure S6. Different relative peak intensities in PXRD result from different degrees of preferential alignment on the substrate. For example, panel A compares two different PXRD samples which show different intensity patterns; one sample (blue) shows a near-random particle distribution, while the other sample (red) has more nanorods, on average, lying parallel to the substrate. Panel B and C show calculated reference patterns for random orientation (B) and partial orientation with the $c$ axis parallel to the substrate (C). 

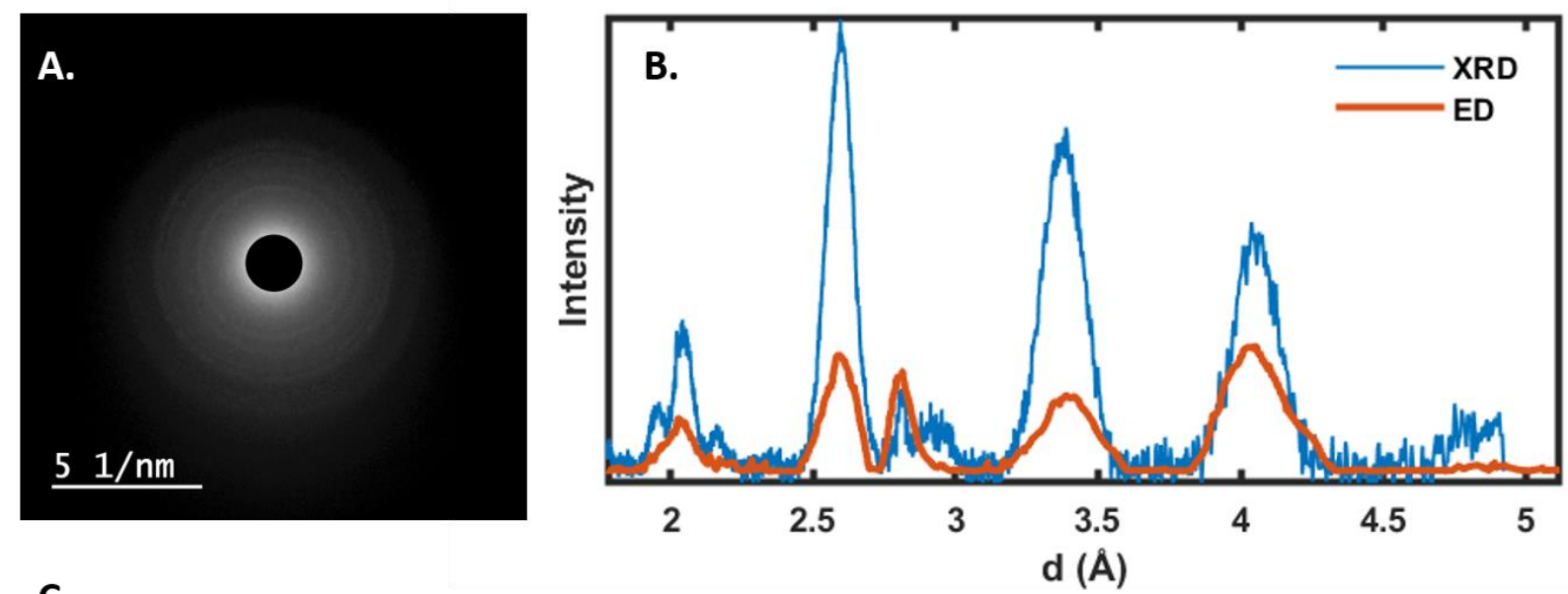

C.

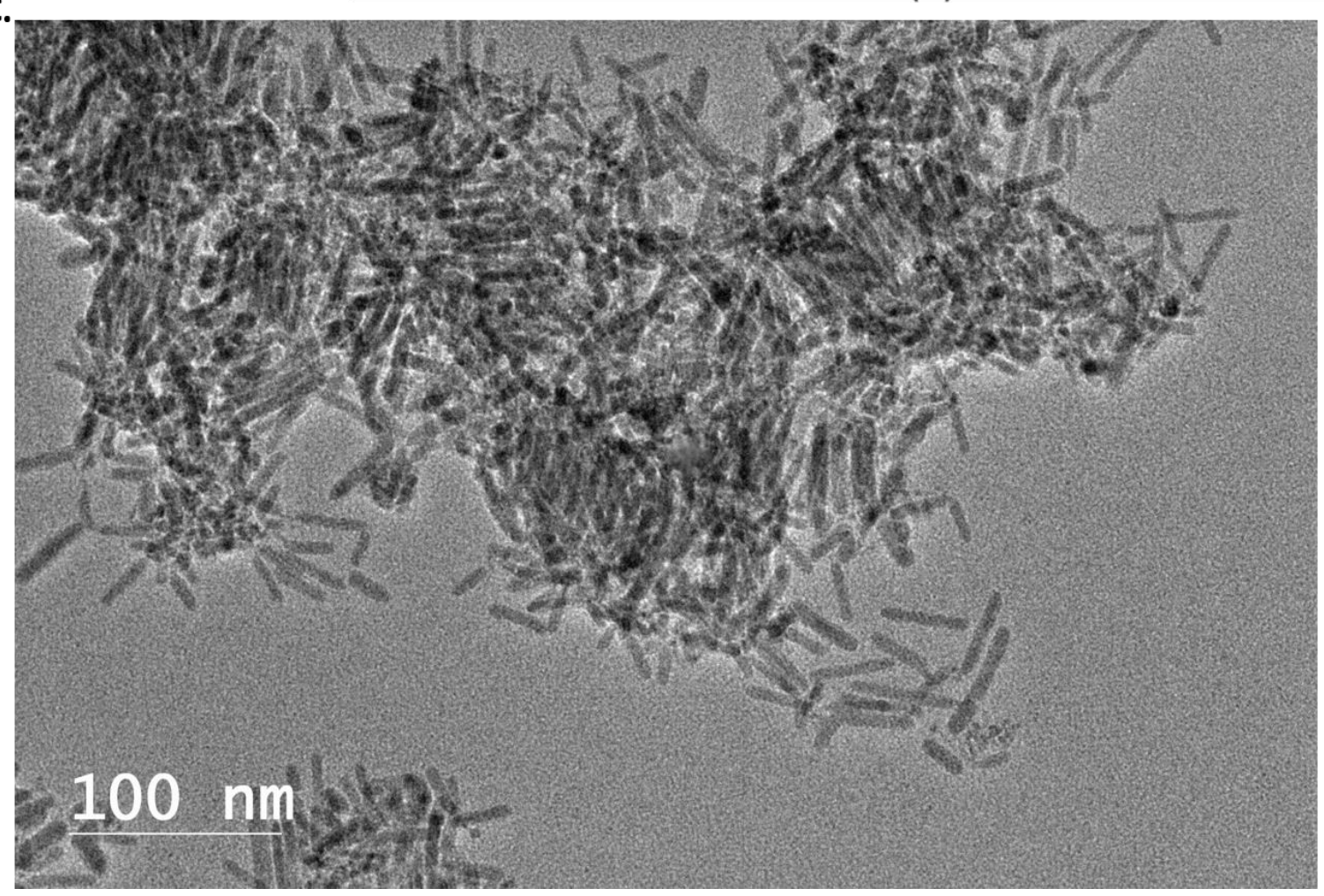

Figure S7. Electron diffraction of a representation $\mathrm{BaTiS}_{3}$ nanorod sample. The observed electron diffraction pattern is shown in (A) with a mask over the center beam spot. Panel (B) shows an overlay of the integrated electron diffraction data overlaid with the powder XRD pattern measured for the same sample, showing good agreement. (C) TEM image of this sample.

\section{Table of Samples and Conditions for Nanorods (hot-injection synthesis).}

The table shown below gives a comprehensive listing of the hot-injection samples tabulated in the scatter plot in Figure 12 in the main text, the conditions used for their synthesis, and other characterization data where available. Further data for some of these samples is shown below. 
Table S1. Synthetic Conditions and Properties for Samples Shown in Figure 12

\begin{tabular}{|c|c|c|c|c|c|c|c|c|c|}
\hline & $\mathrm{T}\left({ }^{\circ} \mathrm{C}\right)$ & $\begin{array}{c}\text { Time } \\
\text { (min. } \\
\text { ) }\end{array}$ & $\begin{array}{c}\text { Stoich. } \\
\text { (Ba:Ti:S) }^{\text {a }}\end{array}$ & $\begin{array}{c}\text { Solvent } \\
\text { Mass } \\
(\mathrm{g})^{\mathbf{b}}\end{array}$ & $\begin{array}{c}\text { Width } \\
\text { (nm) }\end{array}$ & $\begin{array}{c}\text { Length } \\
(\mathrm{nm})\end{array}$ & $\begin{array}{c}\text { NIR } \\
\text { Absorbance } \\
\lambda_{\max }(\mathrm{nm}) \\
\end{array}$ & $\begin{array}{c}(002) \\
\text { reflection } 2 \theta^{f}\end{array}$ & $\begin{array}{c}\text { Apparent } c \\
\text { parameter }(\AA \AA \AA) g\end{array}$ \\
\hline$A$ & 360 & 5 & $1: 1: 30$ & 3.0 & $6.7^{\mathrm{c}}$ & $45^{c}$ & 1713 & $30.94 \pm 0.12$ & $5.76 \pm 0.02$ \\
\hline $\mathrm{B}$ & 360 & 10 & $1: 1: 30$ & 3.0 & $6.8^{c}$ & $40^{c}$ & 1646 & $31.03 \pm 0.09$ & $5.75 \pm 0.02$ \\
\hline $\mathrm{C}$ & 360 & 120 & $1: 1: 30$ & 4.0 & $6.9^{c}$ & $86^{c}$ & 1718 & $31.16 \pm 0.03$ & $5.766 \pm 0.005$ \\
\hline $\mathrm{D}$ & 360 & 120 & $1: 1: 30$ & 3.0 & $15^{c}$ & $96^{c}$ & 1643 & $31.20 \pm 0.02$ & $5.727 \pm 0.004$ \\
\hline$E$ & 360 & 120 & $1: 1: 30$ & 3.0 & $6^{d}$ & e & 1571 & $31.15 \pm 0.04$ & $5.736 \pm 0.006$ \\
\hline $\mathrm{F}$ & 360 & 240 & $1: 1: 30$ & 3.0 & $10^{c}$ & $87^{c}$ & 1648 & $31.51 \pm 0.05$ & $5.697 \pm 0.007$ \\
\hline $\mathrm{G}$ & 360 & 120 & $1: 1: 30$ & 3.0 & $6.5^{\mathrm{c}}$ & $58^{c}$ & 1620 & $31.52 \pm 0.04$ & $5.660 \pm 0.006$ \\
\hline $\mathrm{H}$ & 360 & 15 & $1: 1: 30$ & 3.0 & $6.3^{c}$ & $41^{\mathrm{c}}$ & 1605 & $31.58 \pm 0.06$ & $5.69 \pm 0.01$ \\
\hline $\mathrm{I}$ & 360 & 30 & $1: 1: 30$ & 6.0 & $11^{\mathrm{c}}$ & $28^{c}$ & 1425 & $31.68 \pm 0.02$ & $5.656 \pm 0.004$ \\
\hline $\mathrm{J}$ & 360 & 60 & 1:1.05:30 & 3.5 & $6.0^{c}$ & $50^{c}$ & 1516 & $31.65 \pm 0.06$ & $5.639 \pm 0.009$ \\
\hline $\mathrm{K}$ & 360 & 30 & $1: 1: 30$ & 3.0 & $6.1^{\mathrm{c}}$ & $43^{c}$ & 1447 & $31.73 \pm 0.04$ & $5.643 \pm 0.006$ \\
\hline $\mathrm{L}$ & 360 & 120 & $1: 1.05: 30$ & 3.5 & $6.6^{c}$ & $38^{c}$ & 1496 & $31.77 \pm 0.03$ & $5.618 \pm 0.005$ \\
\hline $\mathrm{M}$ & 360 & 240 & $1: 1.05: 30$ & 3.5 & $8^{d}$ & $\mathrm{e}$ & 1361 & $31.80 \pm 0.03$ & $5.630 \pm 0.005$ \\
\hline $\mathrm{N}$ & 360 & 30 & $1: 1: 30$ & 4.0 & $7.2^{\mathrm{c}}$ & $33^{c}$ & 1370 & $32.11 \pm 0.05$ & $5.574 \pm 0.008$ \\
\hline $\mathrm{O}$ & 360 & $\begin{array}{l}14 \\
\text { hour } \\
\mathrm{s} \\
\end{array}$ & $1: 1: 30$ & 3.0 & $14^{c}$ & $98^{c}$ & 1662 & $31.26 \pm 0.02$ & $5.688 \pm 0.004$ \\
\hline $\mathrm{P}$ & 360 & 5 & $1: 1: 30$ & 3.0 & $5.6^{c}$ & $29^{c}$ & 1674 & $31.10 \pm 0.07$ & $5.72 \pm 0.01$ \\
\hline$Q$ & 360 & 10 & $1: 1: 30$ & 3.0 & $8.0^{c}$ & $19^{c}$ & 1535 & $31.10 \pm 0.07$ & $5.72 \pm 0.01$ \\
\hline $\mathrm{R}$ & 360 & 30 & $1: 1: 30$ & 4.0 & $8^{d}$ & $\mathrm{e}$ & 1635 & $31.40 \pm 0.03$ & $5.650 \pm 0.004$ \\
\hline$S$ & 330 & 120 & $1: 1: 30$ & 3.0 & $6^{d}$ & e & 1546 & $31.64 \pm 0.06$ & $5.63 \pm 0.01$ \\
\hline $\mathrm{T}$ & 280 & 120 & $1: 1: 30$ & 3.0 & $6.8^{c}$ & $63^{c}$ & 1698 & $31.82 \pm 0.02$ & $5.731 \pm 0.004$ \\
\hline$U$ & 360 & 30 & $1: 1: 30$ & 5.0 & $8.7^{c}$ & $15^{\mathrm{c}}$ & 1395 & $31.22 \pm 0.02$ & $5.633 \pm 0.004$ \\
\hline
\end{tabular}

${ }^{\mathrm{a}} 1$ equivalent corresponds to $0.1 \mathrm{mmol}$ of the precursor. ${ }^{\mathrm{b}}$ Solvent is oleylamine. ${ }^{\mathrm{C}}$ Measured by TEM; represents the average of the measurement of at least 100 particles. ${ }^{d}$ Estimated from PXRD using the Scherrer equation. ${ }^{\text {Not }}$ available. ${ }^{f}$ Corrected for sample displacement error as determined by Rietveld refinement (vide infra) Errors shown are based on the uncertainty in the determination of the sample displacement error. ${ }^{~}$ As determined by Rietveld refinement (vide infra). Errors shown are based on the uncertainty in the determination of $c$.

\section{ICP-MS Data for Nanoparticles and Nanorods}

Table S2: Stoichiometry (Ba:Ti) determined by ICP-MS for nanocrystals synthesized at $360^{\circ} \mathrm{C}$ using either heat-up or hot-injection methods and different reaction stoichiometries.

\begin{tabular}{|l|l|l|l|}
\hline Sample & Method & $\begin{array}{l}\text { Stoichiometry in } \\
\text { Reaction (Ba:Ti:S) }\end{array}$ & $\begin{array}{l}\text { Measured } \\
\text { Stoichiometry (Ba:Ti) }\end{array}$ \\
\hline$T$ & hot injection & $1: 1: 30$ & $1: 1.2$ \\
\hline$U$ & hot injection & $1.2: 1: 30$ & $1: 1.15$ \\
\hline V & hot injection & $1.5: 1: 30$ & $1: 1.4$ \\
\hline W & hot injection & $1: 1: 30$ & $1: 1.44$ \\
\hline$X$ & hot injection & $1: 1: 30$ & $1: 1.61$ \\
\hline Y & hot injection & $1: 1: 30$ & $1: 1.38$ \\
\hline$Z$ & hot injection & $1: 1: 30$ & $1: 1.44$ \\
\hline AA & hot injection & $1: 1: 30$ & $1: 1.33$ \\
\hline$A B$ & heat up & $1: 1: 30$ & $1: 1.05$ \\
\hline
\end{tabular}




\section{Additional TEM Data for Samples Shown in the Main Text}

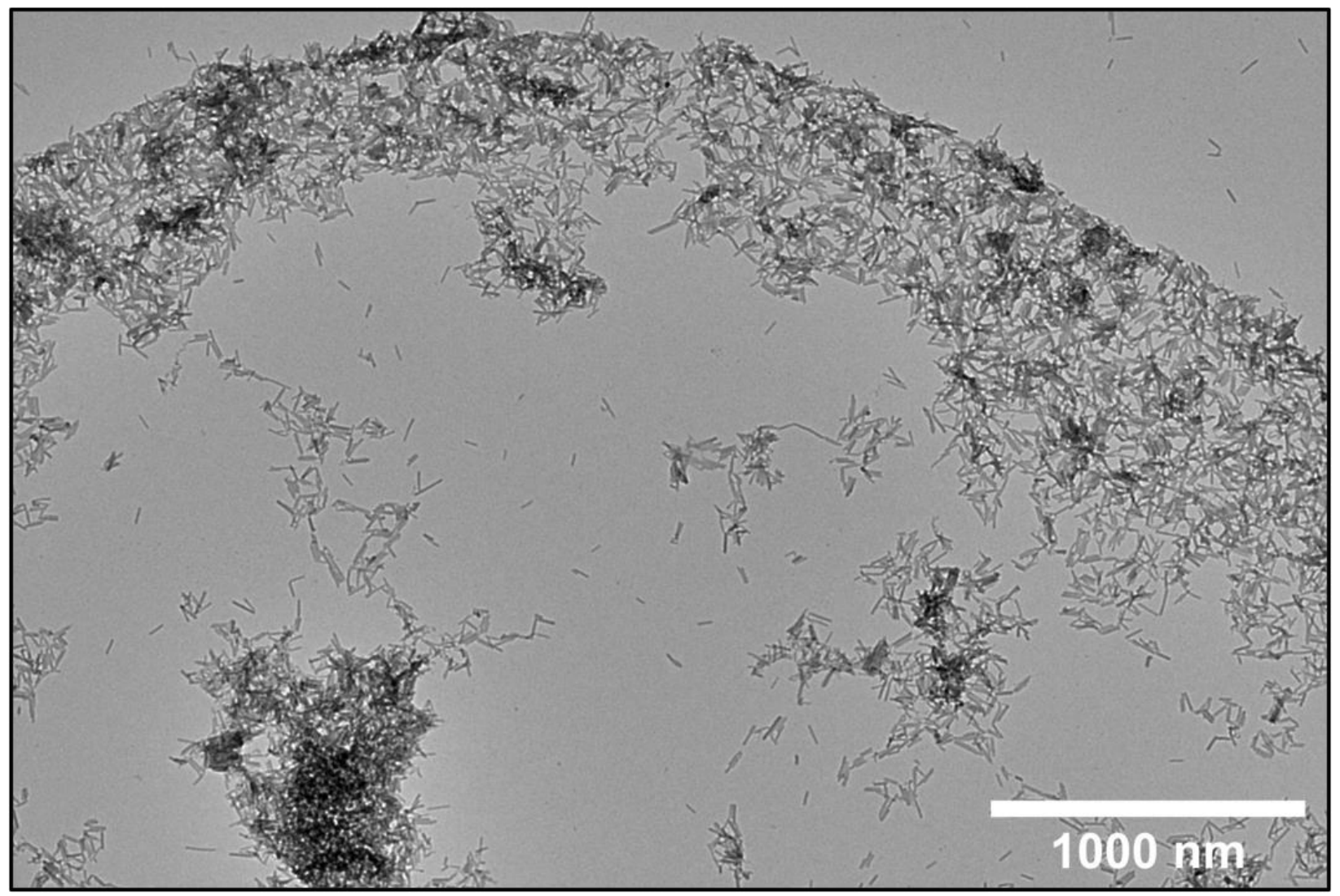

Figure S8. Wide-area TEM image of representative hot-injection nanorods synthesized under standard conditions (data from this sample was shown in Figure 4 of the main text). 


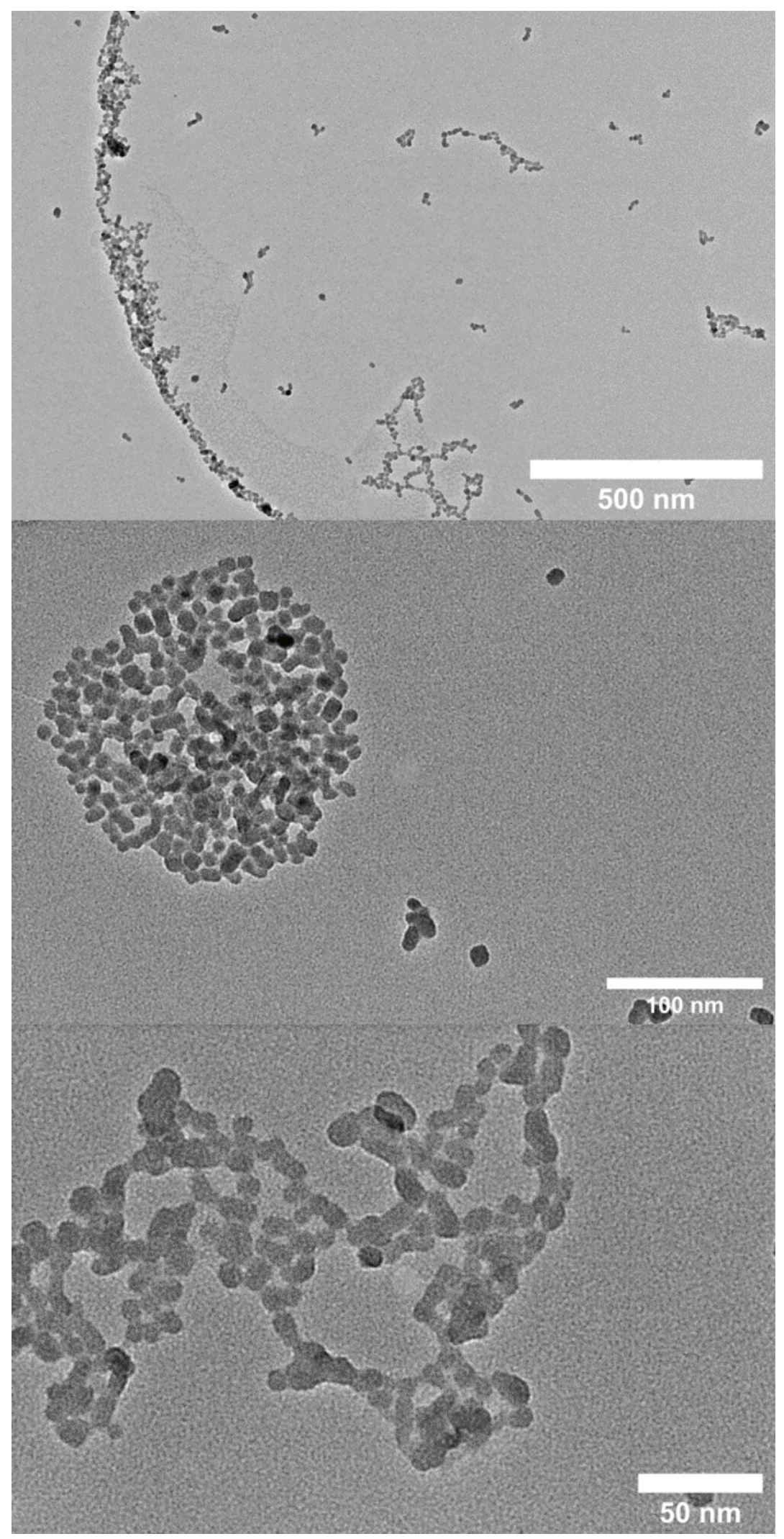

Figure S9: Additional TEM images for the representative heat-up sample shown in the main text (Figure $6)$. 


\section{Synthetic Variations}

Note: Reactions described in this section were carried out using the standard conditions described in the Experimental Methods section of the main text, except for the differences explicitly noted.

Heat-Up Synthesis: Data from a heat-up synthesis using 15 equivalents of the sulfur source, $\mathrm{N}, \mathrm{N}$ '-diethylthiourea, was shown in the main text. Data from an additional sample synthesized with 30 equivalents of the sulfur source are shown here. The samples are generally similar, although the average size of the nanoparticles from the 30 -equivalents reaction is slightly larger (11.7 nm vs. $9.2 \mathrm{~nm}$ ). 

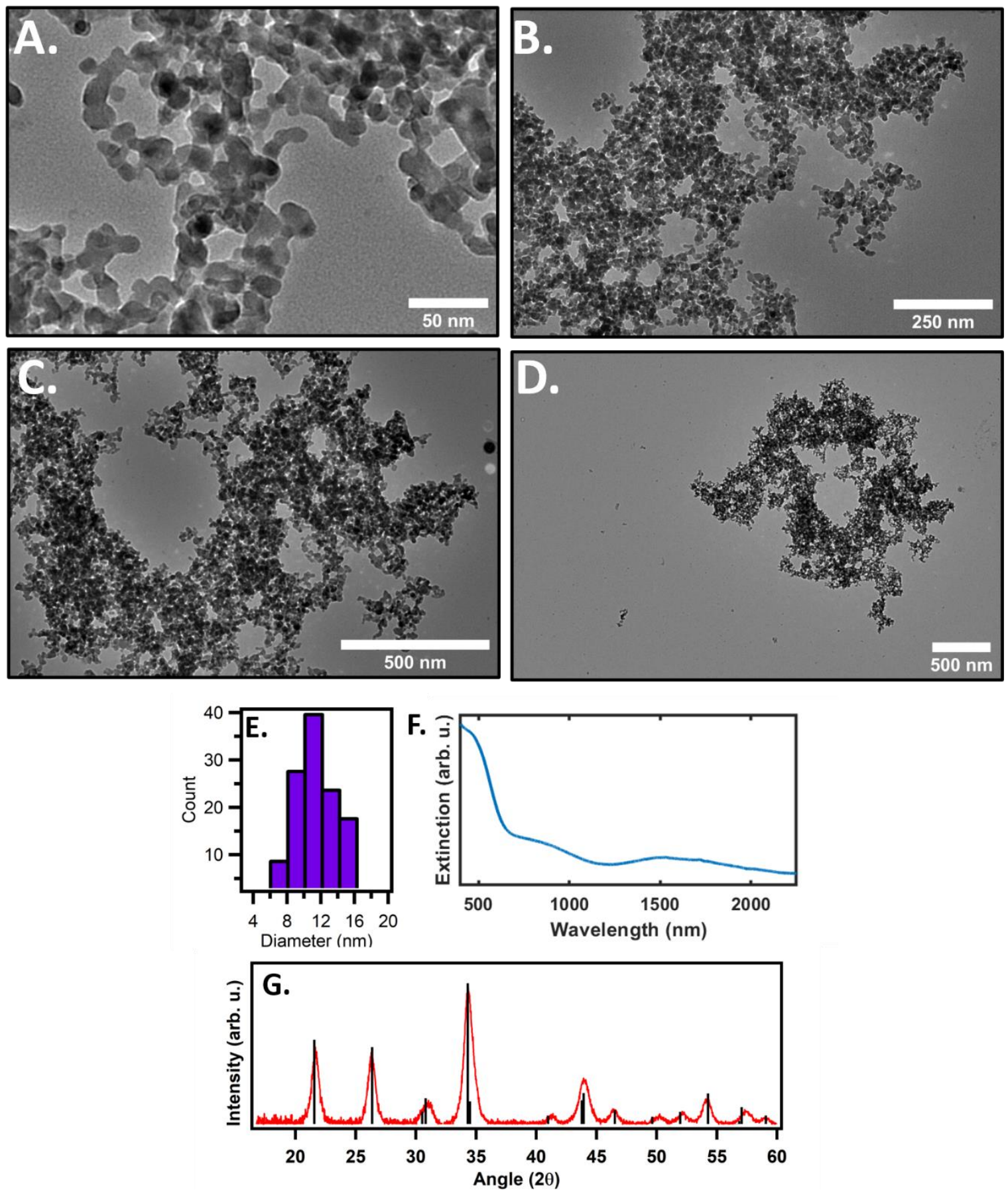

Figure S10. Characterization data for an additional representative sample of nanocrystals synthesized using the heat-up method (1:1 Ba:Ti ratio, 30 equivalents of $\mathrm{N}, \mathrm{N}$ '-diethylthiourea; $3 \mathrm{~g}$ of oleylamine; 360 ${ }^{\circ} \mathrm{C}$; reaction time 2 hours). (A-D) TEM images; scale bars are 50, 250, 500, and $500 \mathrm{~nm}$, respectively. $€$ Histogram of nanoparticle sizes, based on measurements of 125 individual particles; average size is 11.7 $\pm 3.0 \mathrm{~nm}$. (F) UV-Vis-NIR spectra taken in chloroform. (G) PXRD data overlaid with predicted reference pattern of $\mathrm{BaTiS}_{3}$ in $\mathrm{PG}_{3} / \mathrm{mmc}$. 
Heat-up synthesis at short reaction times. The standard reaction time used for the heat-up synthesis was 2 hours. We also tested a reaction with a much shorter reaction time. In this case, the reaction was removed from the heat immediately after reaching $360^{\circ} \mathrm{C}$, then worked up and analyzed following standard methods. Results are shown below. 


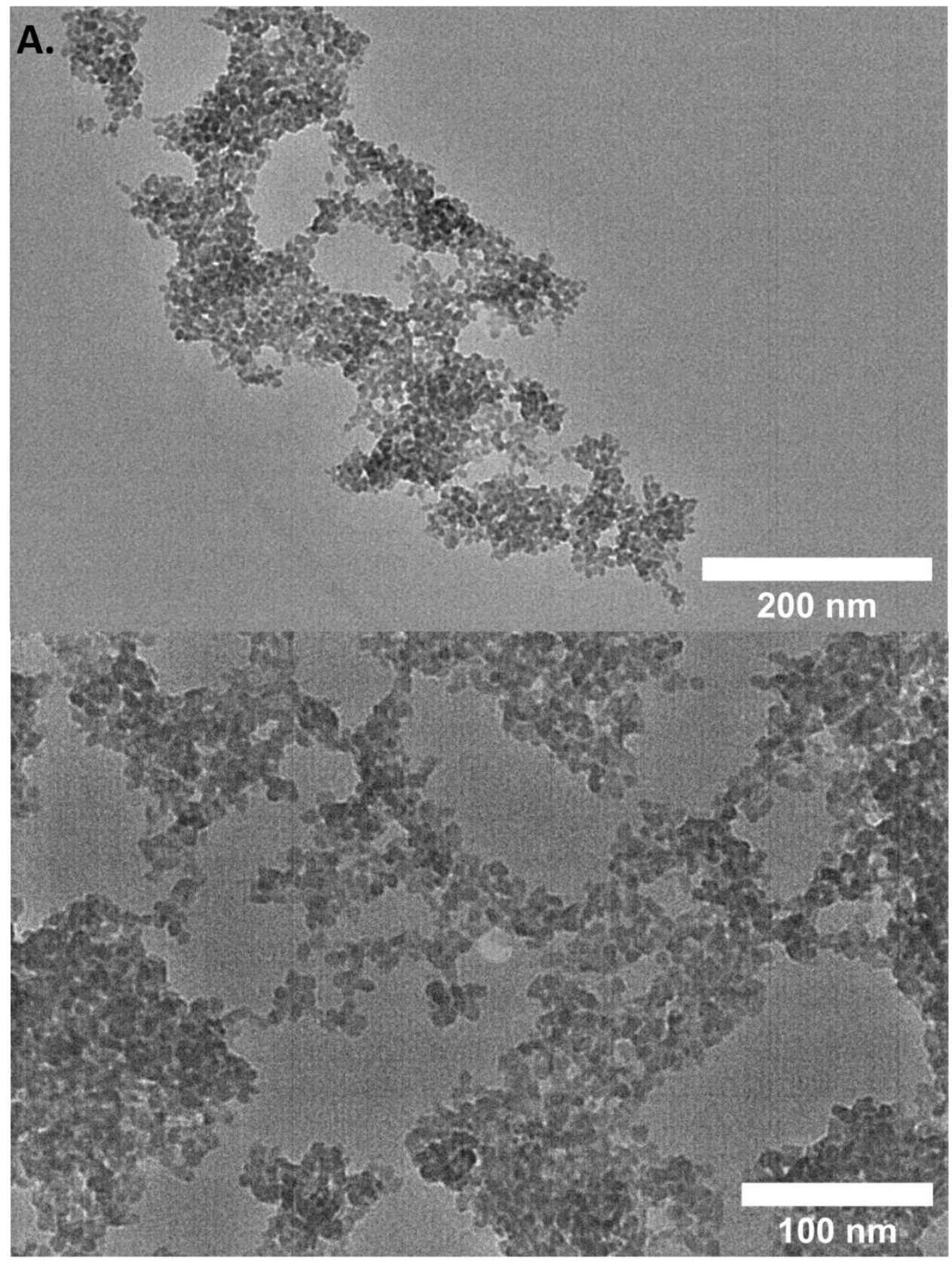

B.
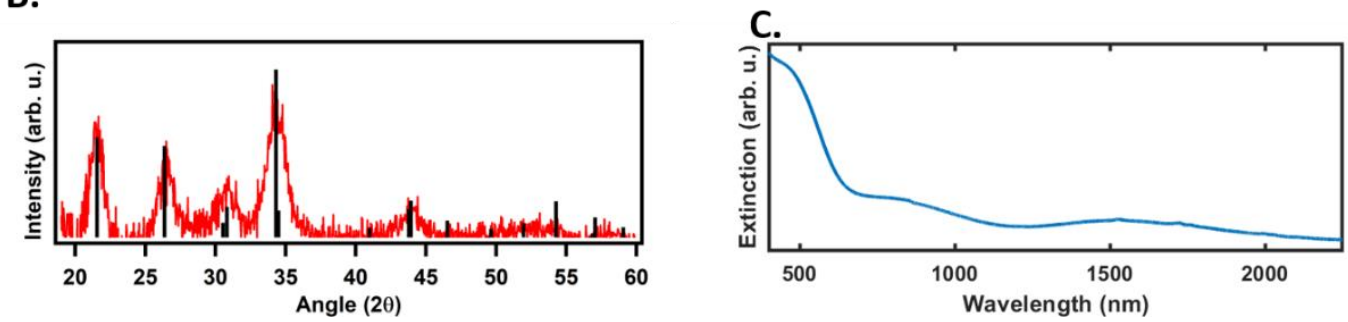

Figure S11. Nanocrystals synthesized using a heat-up protocol at $360^{\circ} \mathrm{C}$ with a "zero minute" reaction time (removed from heat immediately after reaching $360^{\circ} \mathrm{C}$ ). (A) TEM images; (B) PXRD data (red) overlaid with $\mathrm{BaTiS}_{3}$ reference pattern (black); (C) UV-Vis-NIR spectrum. 
Additional Data for Hot-Injection Time Series (Figure 10 in Main Text)

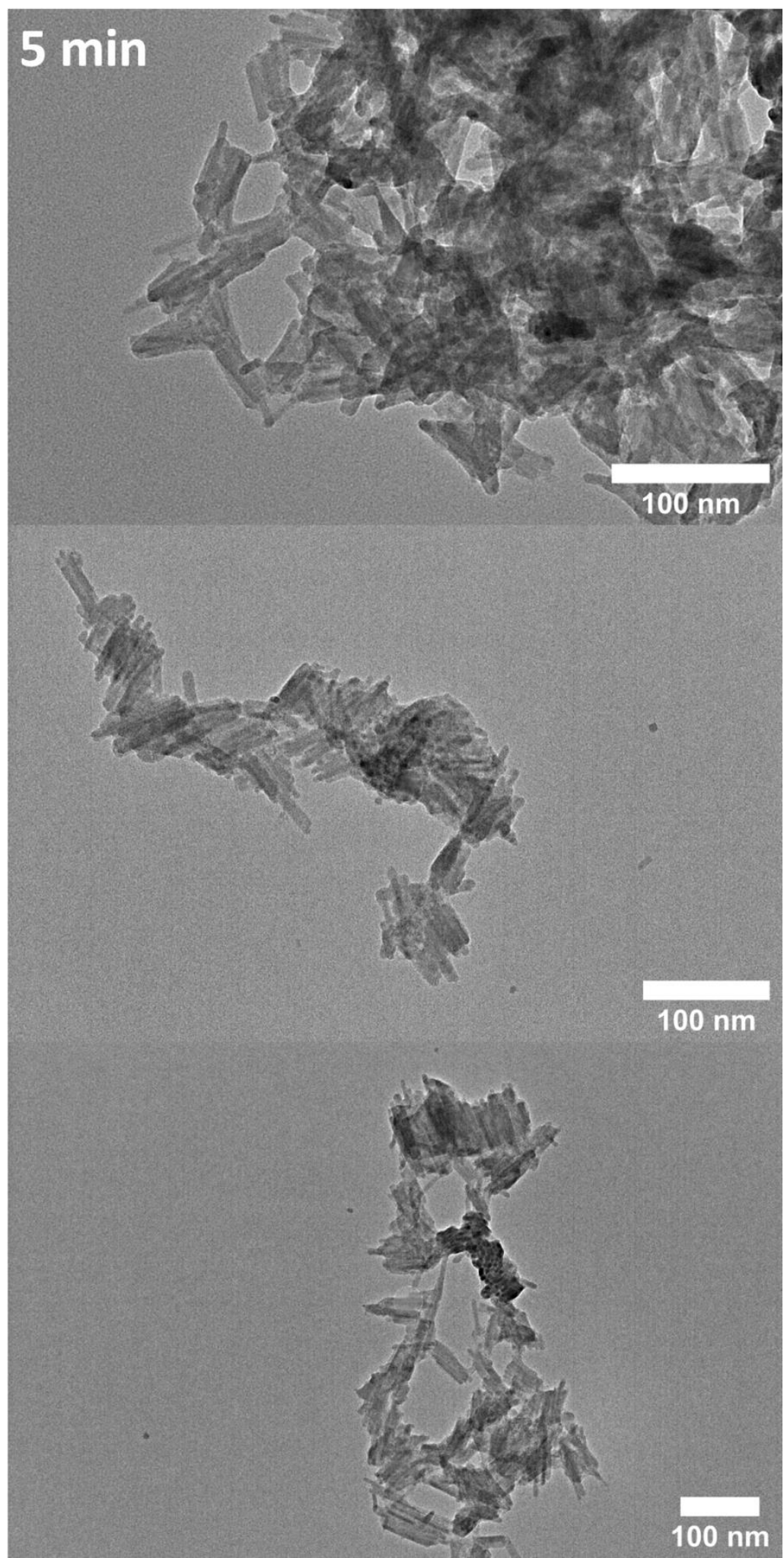

Figure S12. TEM data for aliquots taken from a reaction after 5 minutes, as discussed in the main text. 


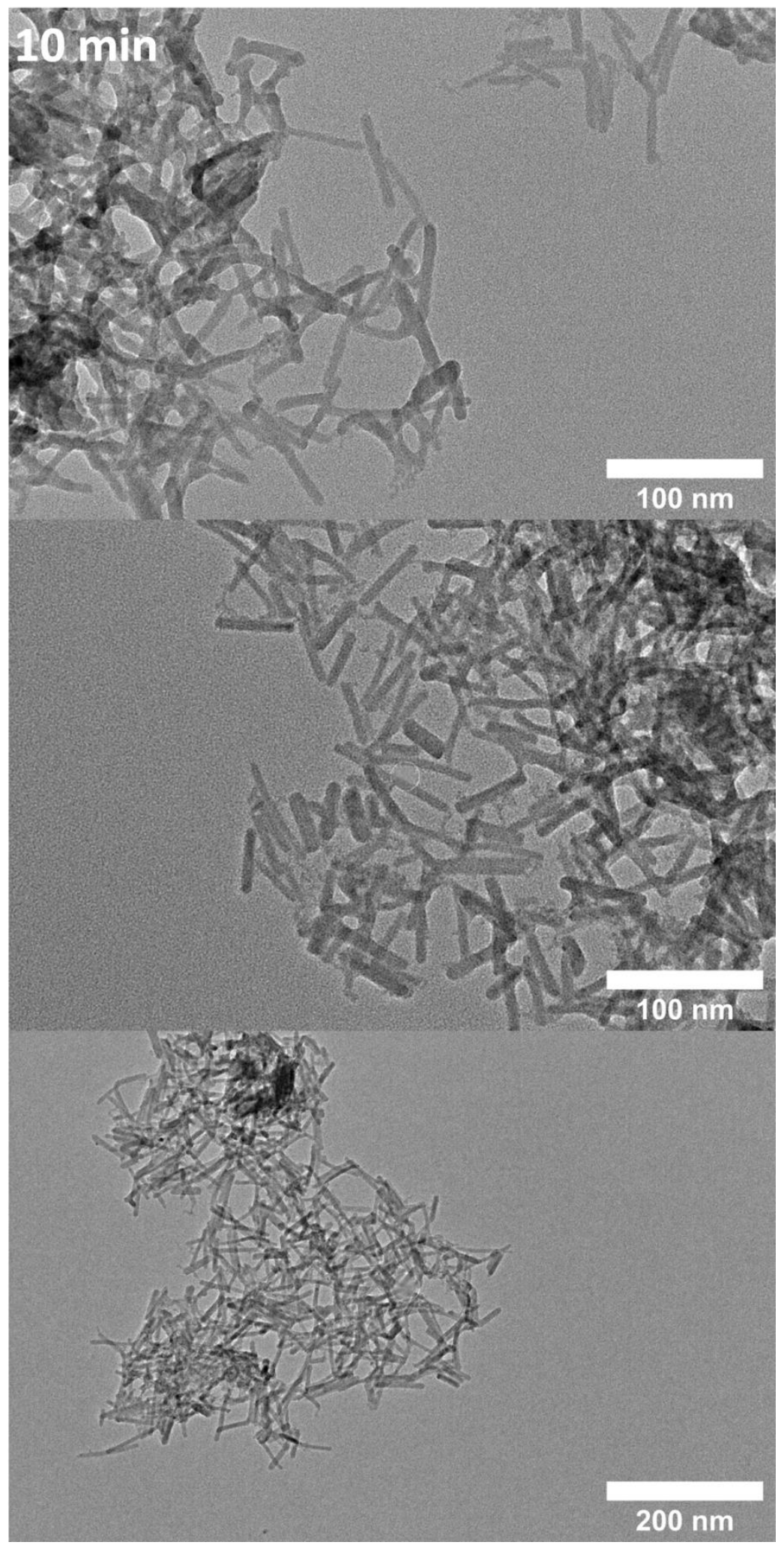

Figure S13. Additional TEM data for aliquots taken from a reaction after 10 minutes, as discussed in the main text. The faint circle that appears at the center of some images is an artefact from the TEM camera. 


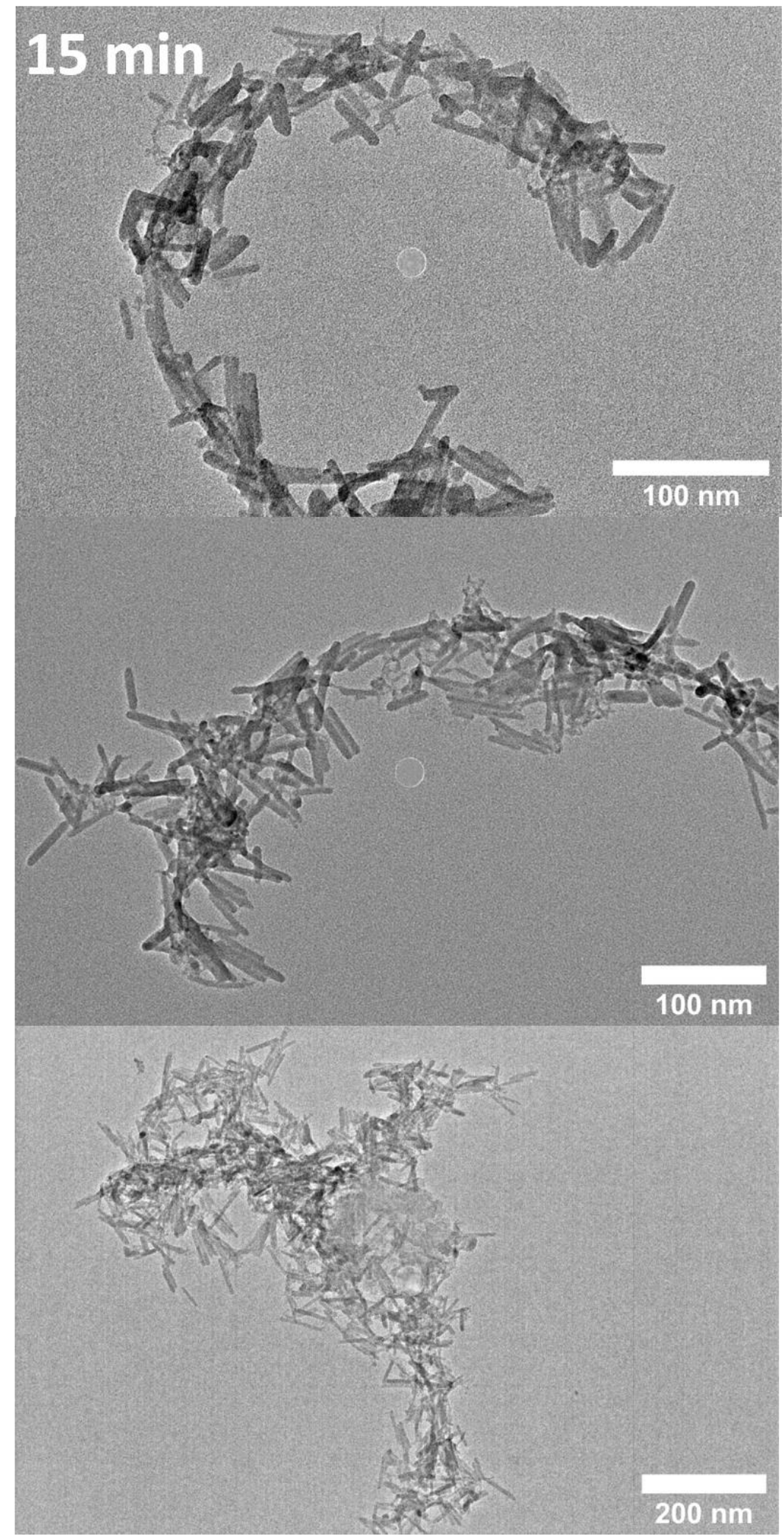

Figure S14. TEM data for aliquots taken from a reaction after 5 minutes, as discussed in the main text. The faint circle that appears at the center of some images is an artefact from the TEM camera. 


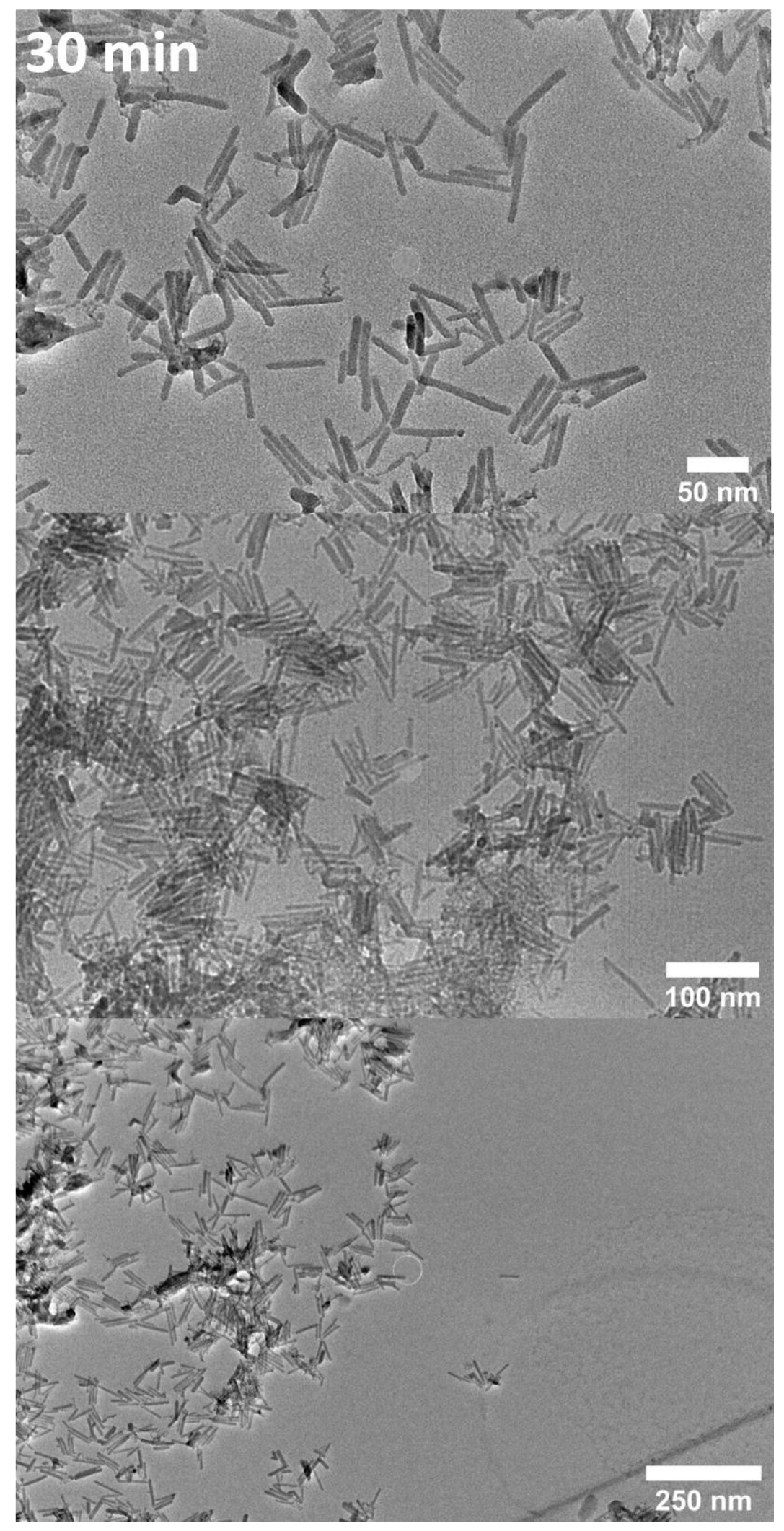

Figure S15. TEM data for aliquots taken from a reaction after 5 minutes, as discussed in the main text. The faint circle that appears at the center of some images is an artefact from the TEM camera. 


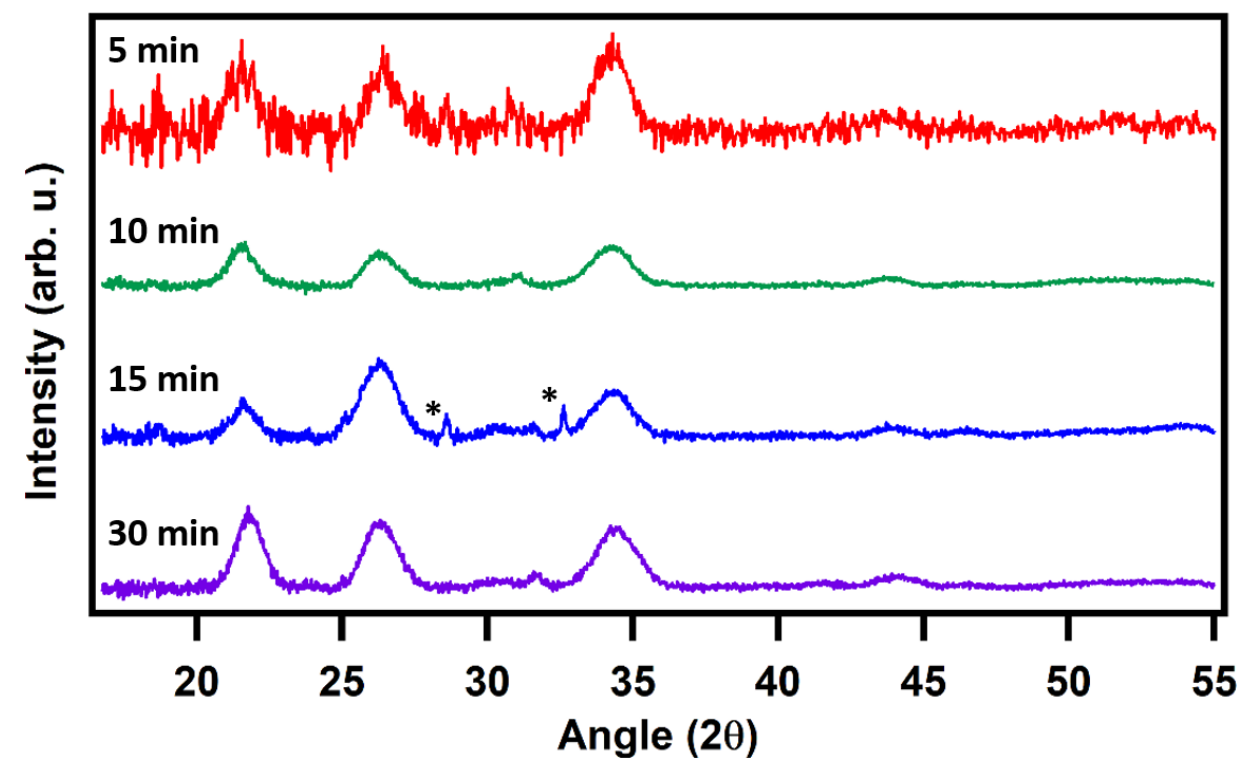

Figure S16. PXRD data for the nanocrystals from the aliquots taken at different times in the reaction shown in Figure 10 in the main text. The identity of the impurity seen in the 15 minute sample $(*)$ is unknown; since it is not present in the other samples, it is most likely something that formed in the sample after removal from the reaction mixture, perhaps due to exposure to air. 
A.
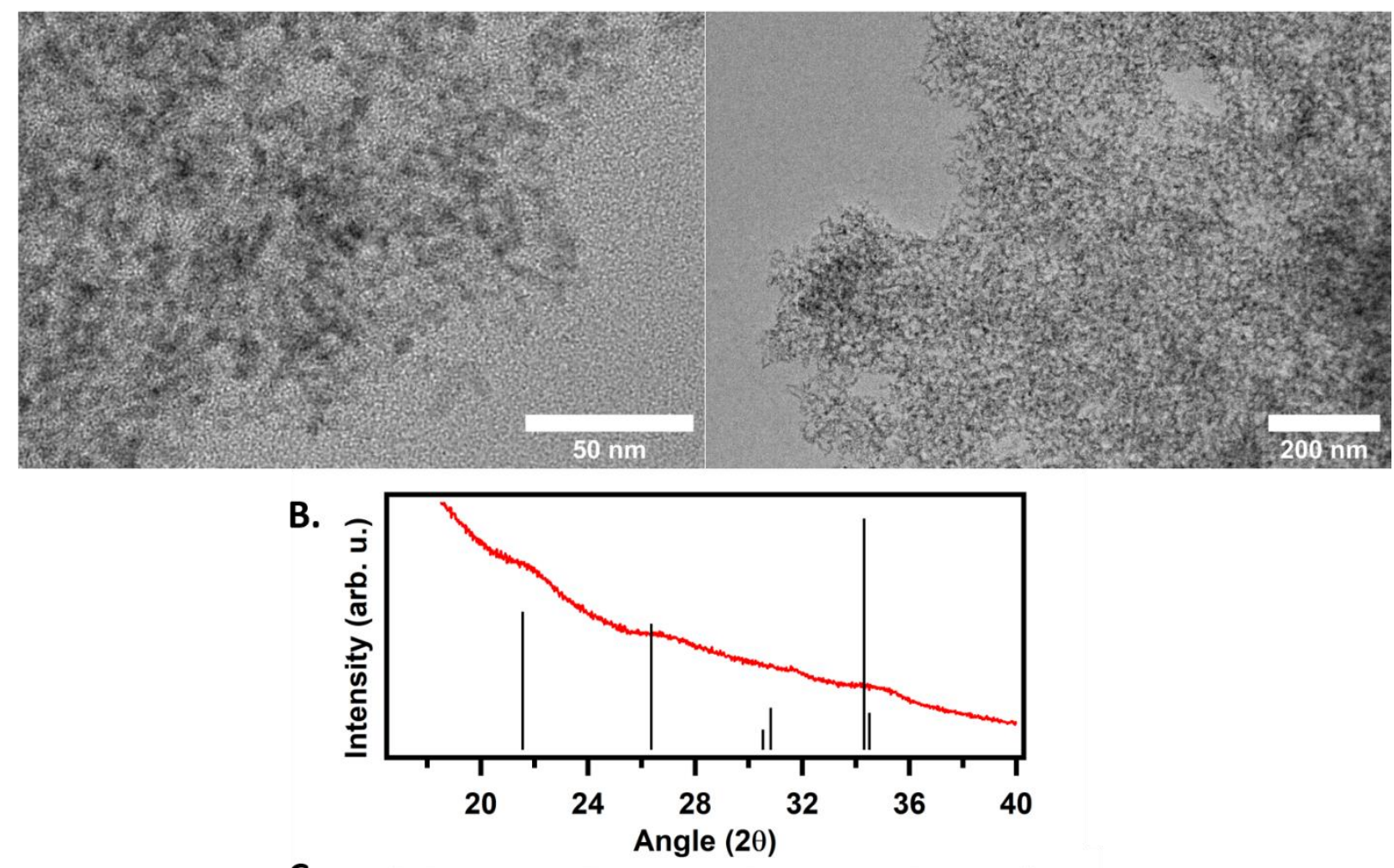

C.

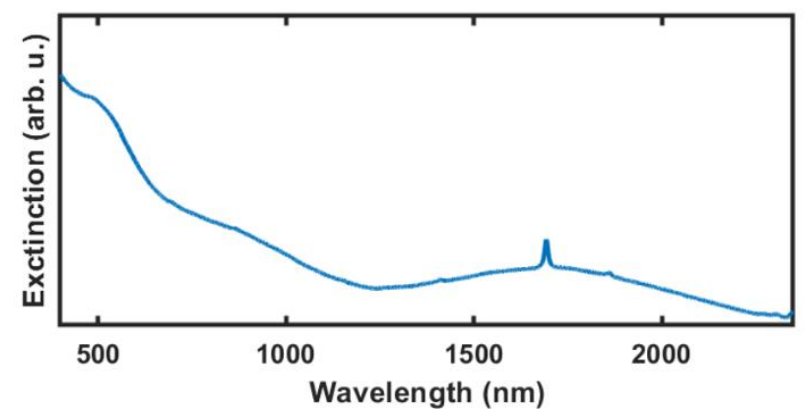

Figure S17. Data for a hot-injection sample synthesized with a "0 minute" reaction time at $360^{\circ} \mathrm{C}$ (reaction mixture was removed from heat immediately after injection of sulfur source). (A) TEM images;

(B) PXRD overlaid with reference pattern for $\mathrm{BaTiS}_{3}$; (C) UV-Vis-NIR spectrum in tetrachloroethylene. This data suggests that, although XRD and optical signatures for the presence of $\mathrm{BaTiS}_{3}$ are observed, slightly longer reaction times are required for the formation of well-defined nanocrystals (see above). 


\section{Variable Temperature Hot-Injection Synthesis}

Reaction temperatures between $250^{\circ} \mathrm{C}$ and $360^{\circ} \mathrm{C}$ were tested. A two hour reaction time was used for temperatures below $360^{\circ} \mathrm{C}$. No BaTiS $S_{3}$ nanoparticles were formed at $250{ }^{\circ} \mathrm{C}$; results from reactions at $280^{\circ} \mathrm{C}, 300^{\circ} \mathrm{C}$, and $330^{\circ} \mathrm{C}$ are shown below. At $280^{\circ} \mathrm{C}$, it was observed that the onset of reaction (as judged by the color change to black) was not immediate upon injection but took several minutes. A reaction was carried out at $280^{\circ} \mathrm{C}$ with a shorter reaction time (30 minutes); at this point, the nanocrystals were not well formed. This data is also shown below. 


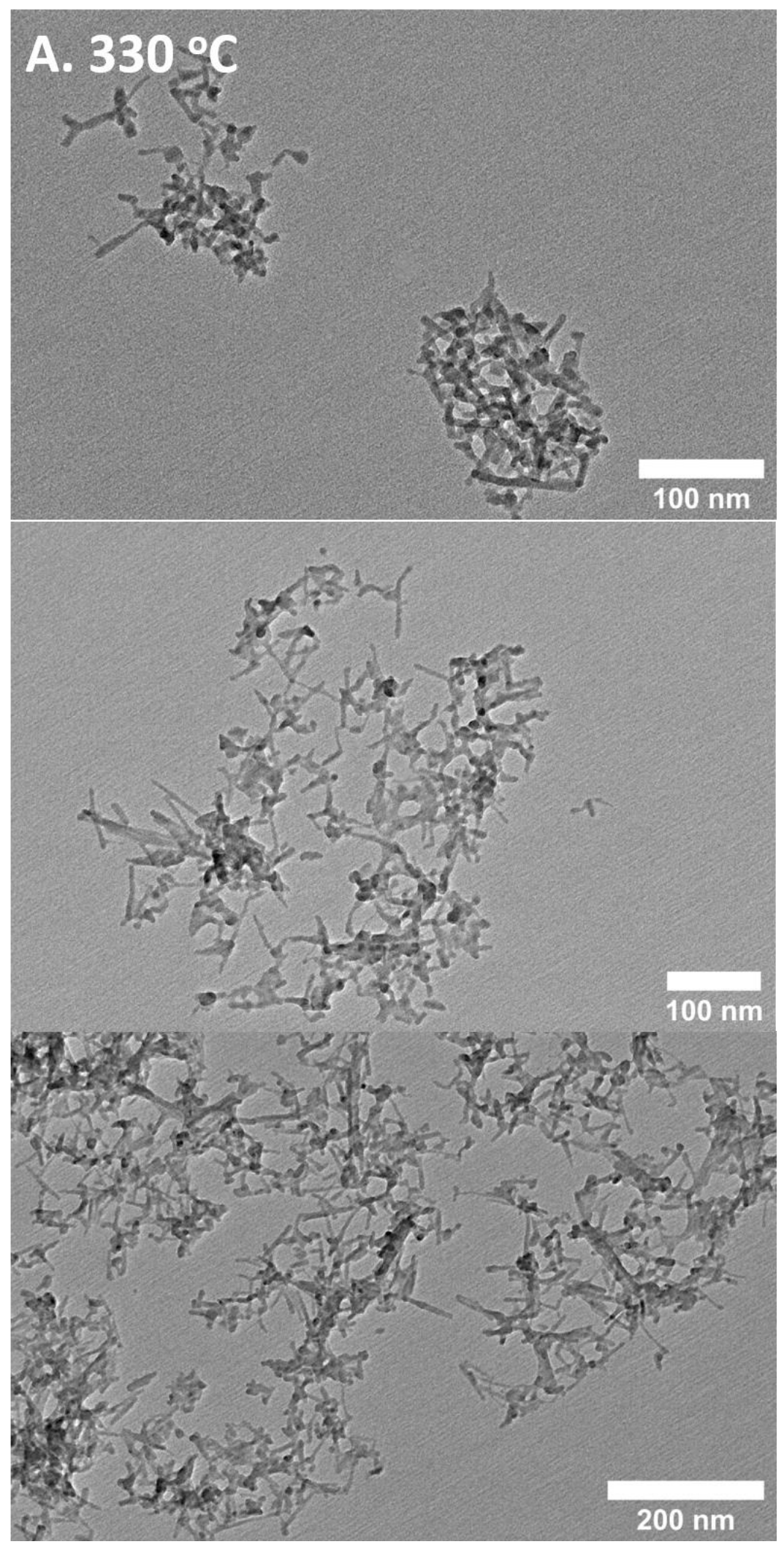

Figure S18. Nanocrystals synthesized at $330^{\circ} \mathrm{C}$. The nanocrystals from this reaction were somewhat irregular in shape, precluding a clear measurement of length vs. width. 


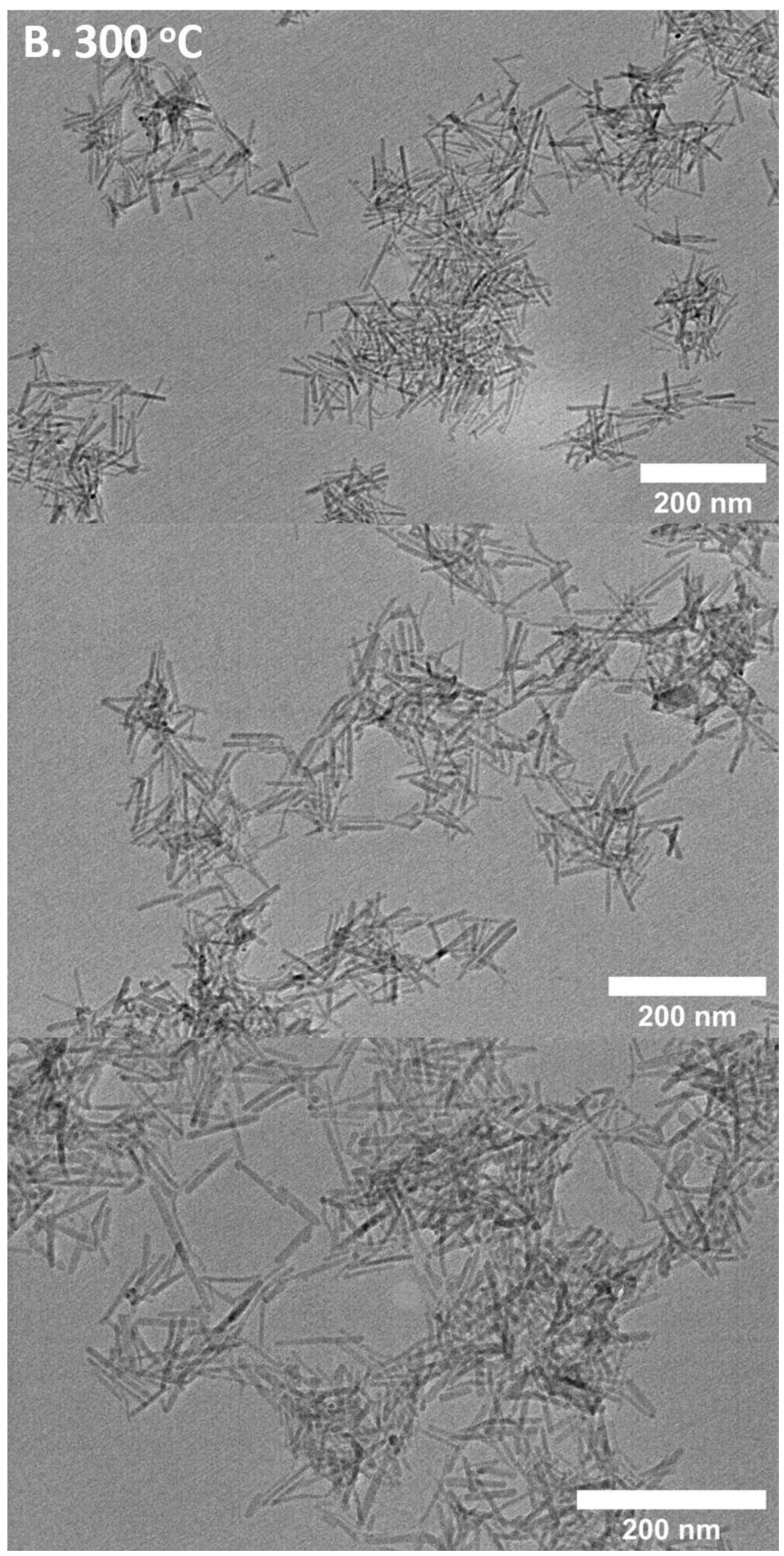

Figure S19. Nanocrystals synthesized at $300^{\circ} \mathrm{C}$. Average width: $6.1 \pm 1.6 \mathrm{~nm}$. Average length: $62 \pm 16$ $\mathrm{nm}$. 


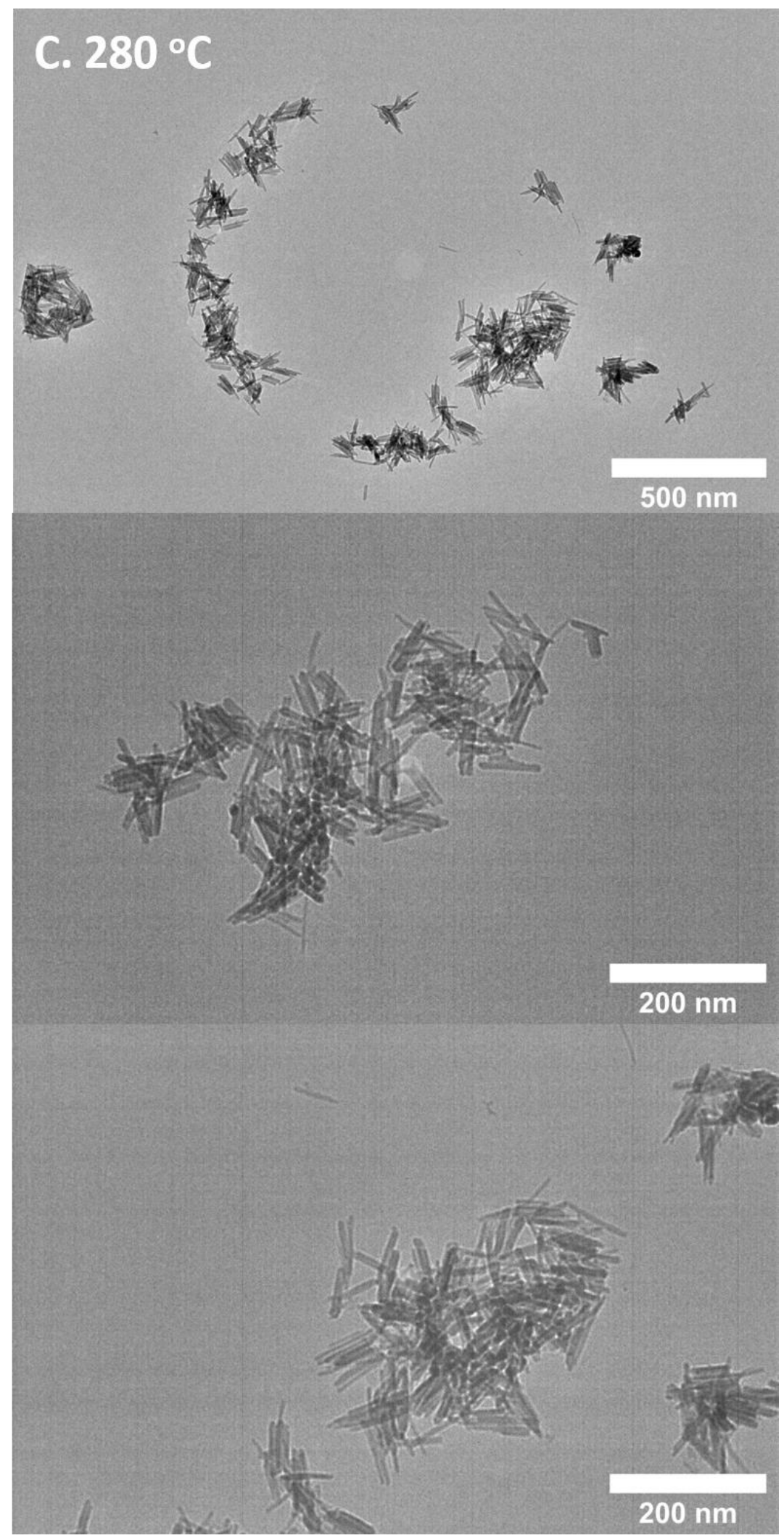

Figure S20. Nanocrystals synthesized at $280^{\circ} \mathrm{C}$. Average width: $6.8 \pm 1.6 \mathrm{~nm}$. Average length: $63 \pm 15$ $\mathrm{nm}$. 


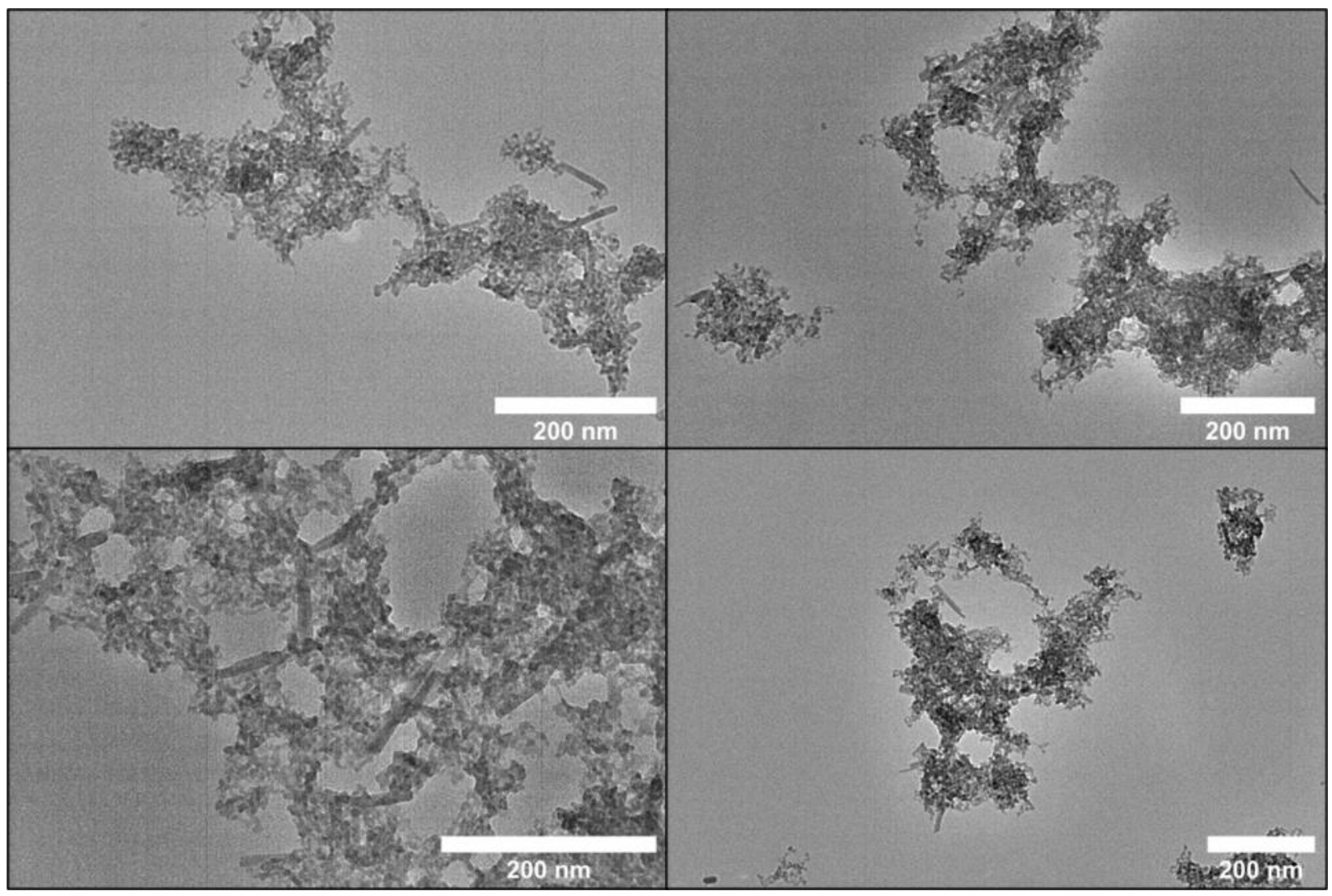

Figure S21. TEM images of material formed from a hot-injection reaction at $280^{\circ} \mathrm{C}$ with a 30 minute reaction time. Although there are a few well-formed nanorods visible, the material is mostly poorly defined and irregular particles. 
A.

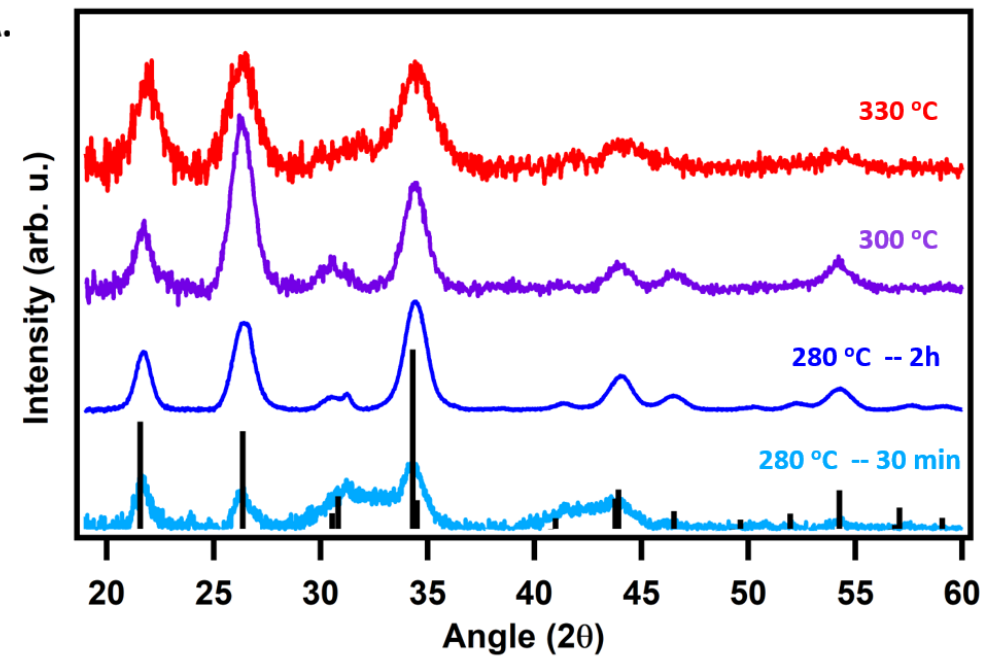

B.

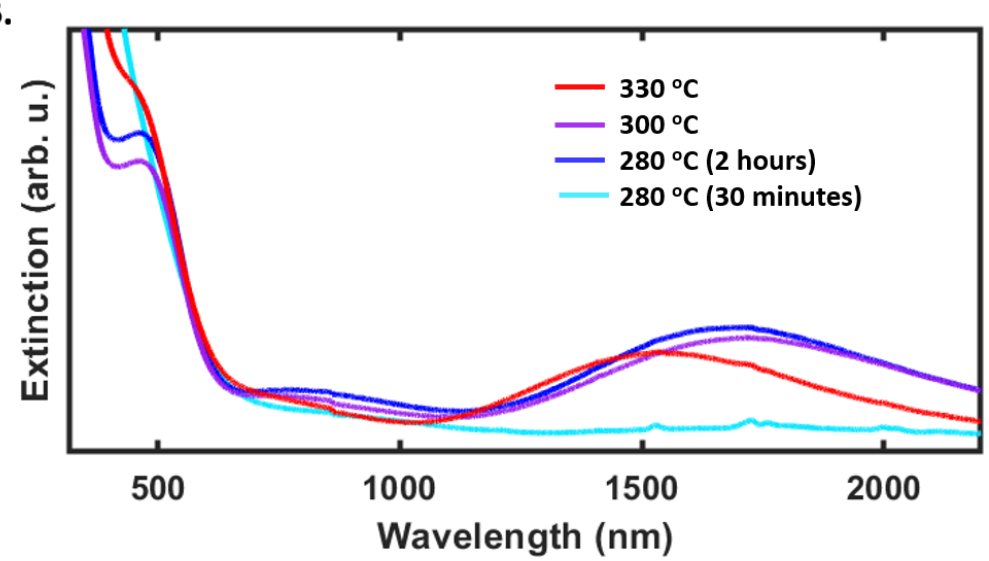

Figure S22. Further characterization for nanocrystals synthesized at different temperatures using a hotinjection approach. Reaction times were 2 hours except where otherwise noted. (A) PXRD data, overlaid with calculated reference pattern for $\mathrm{BaTiS}_{3}$ (black lines); (B) UV-Vis-NIR data, taken in tetrachloroethylene. 
Additional Data for Hot-Injection Nanorods Synthesized at Different Concentrations

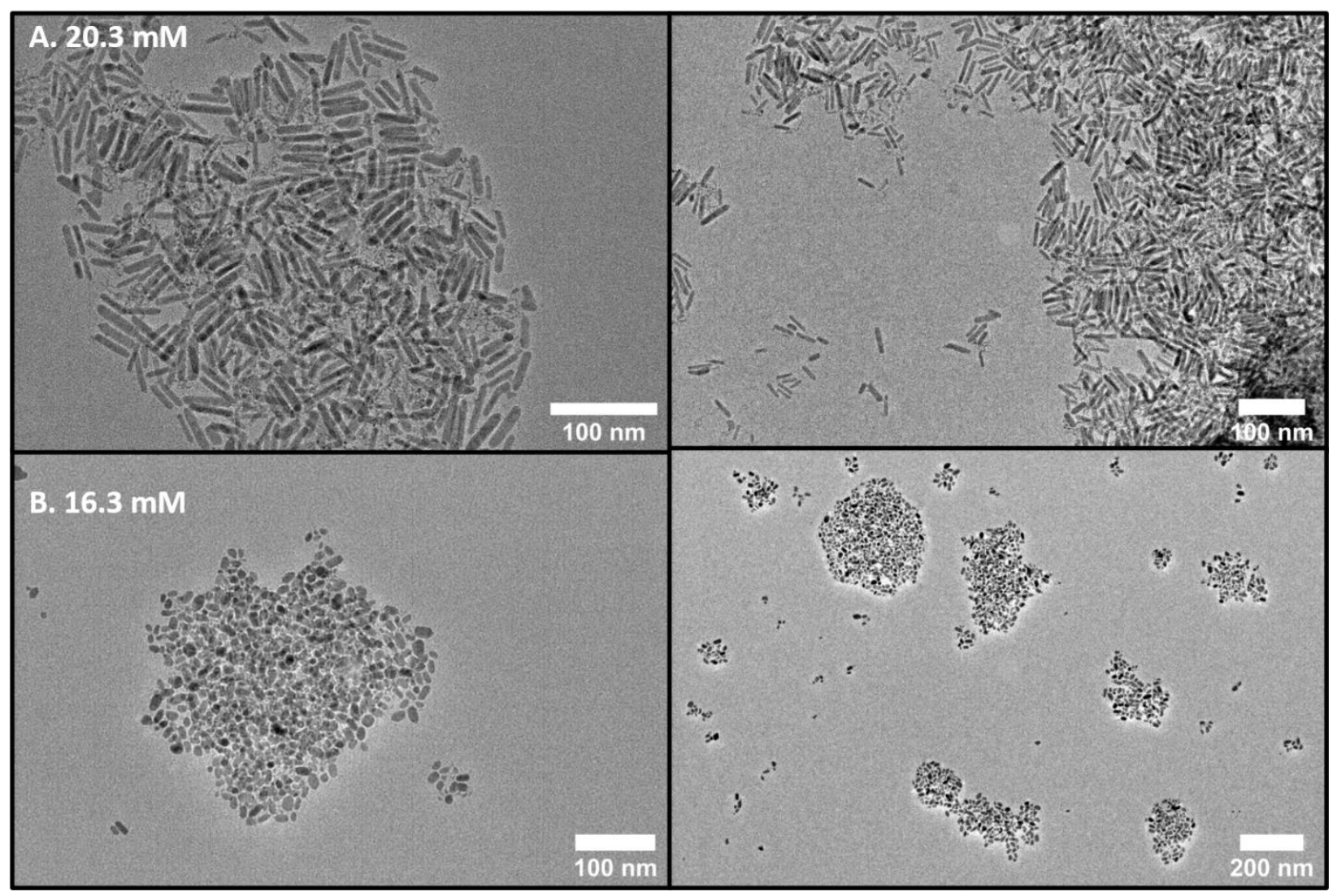

Figure S23. Additional TEM images for nanocrystals synthesized by hot injection at $20.3 \mathrm{mM}$ or $16.3 \mathrm{mM}$ metal precursor concentrations. 


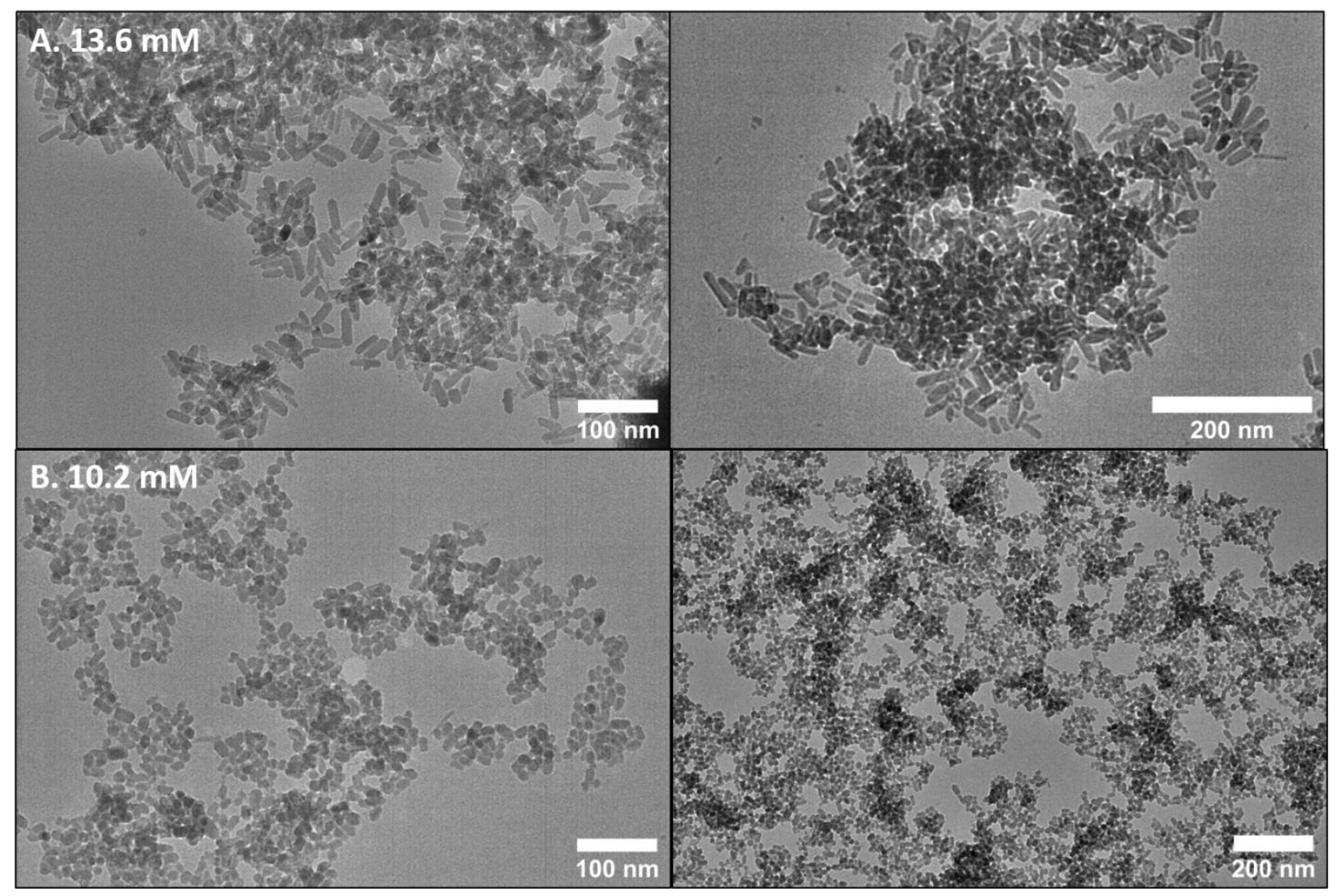

Figure S24. Additional TEM images for nanocrystals synthesized by hot injection at $13.6 \mathrm{mM}$ or $10.2 \mathrm{mM}$ metal precursor concentrations. 

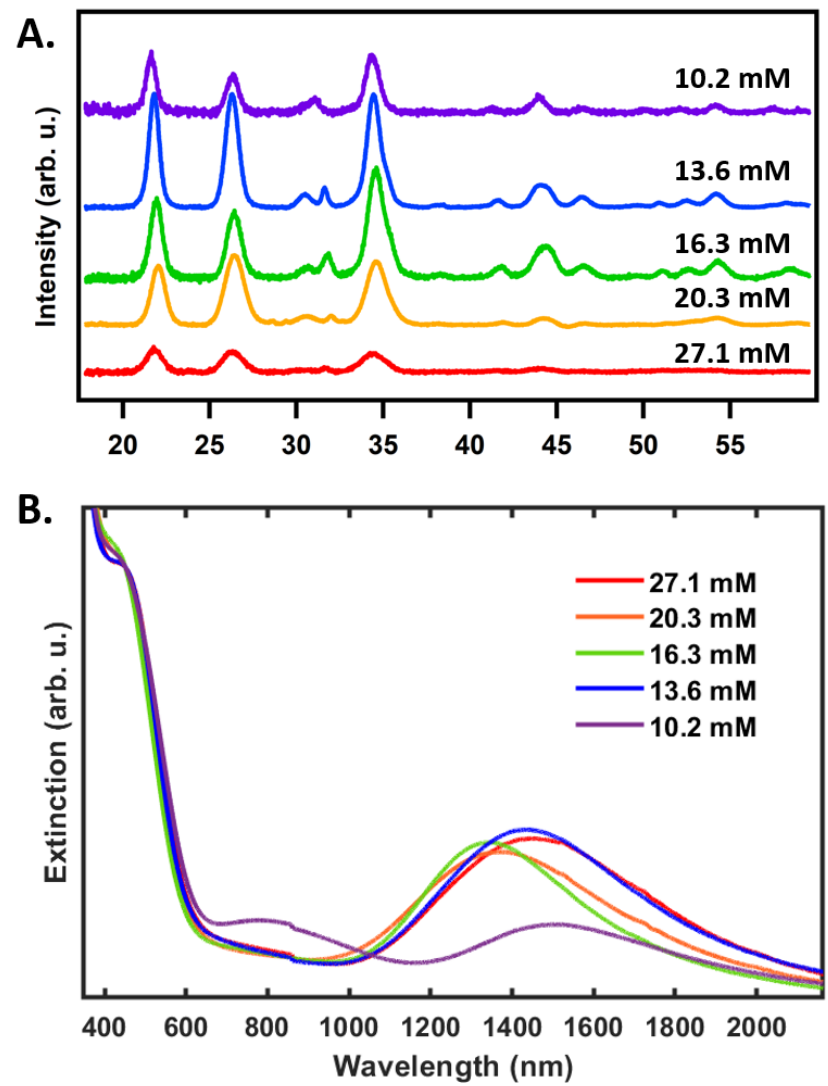

Figure S25. PXRD (A) and UV-Vis-NIR (B) data for nanorods synthesized by hot injection at different metal precursor concentrations. TEM and size data for these samples was shown in the text. 


\section{FWHM of Sample PXRD Over Time}

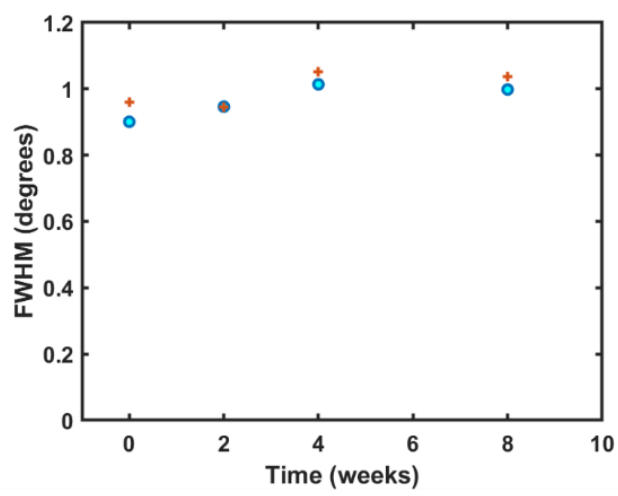

Figure S26. Plot of the FWHM of the peaks at $22^{\circ}$ (blue) and $26^{\circ}$ (orange) in the PXRD of BaTiS 3 plotted as a function of time, derived from the data shown in Figure $9 \mathrm{~A}$ of the main text based on fits of the peaks to Voigt-shape functions.

\section{Rietveld Refinement of Nanocrystal PXRD.}

Refinement was carried out using Jade 9 with the structural data from Z. Naturforsch. B 1980, 35, 775 in $P 6_{3} / m m c$ used as the starting point for the refinement. Lattice parameters $a$ and $c$ were refined; atomic coordinates and occupancies were not refined and were fixed the published values and at 1.0, respectively. The sample displacement error was refined freely. A reference scan of an $\mathrm{LaB}_{6}$ standard was used to determine the instrument parameters for peak fitting. Peak profiles were pseudo-Voigt, and anisotropic broadening was fit using $4^{\text {th }}$-order spherical harmonics. Preferred orientation was included using the March-Dollase model with alignment along [001] and the March-Dollase parameter was refined. Several examples of representative data are shown below. Poorly-matched regions of the fit are hypothesized to originate from unmodeled factors such as (i) the composite modulated structure of $\mathrm{BaTiS}_{y}{ }^{6}{ }^{6}$ (ii) non-stoichiometry (e.g., partial occupancy of $\mathrm{S}$ and/or $\mathrm{Ba}$ ); (iii) other deviations from the bulk structure, including those due to surface effects; (iv) the small finite size of the nanocrystals. 
Nanorod Sample, Example \#1
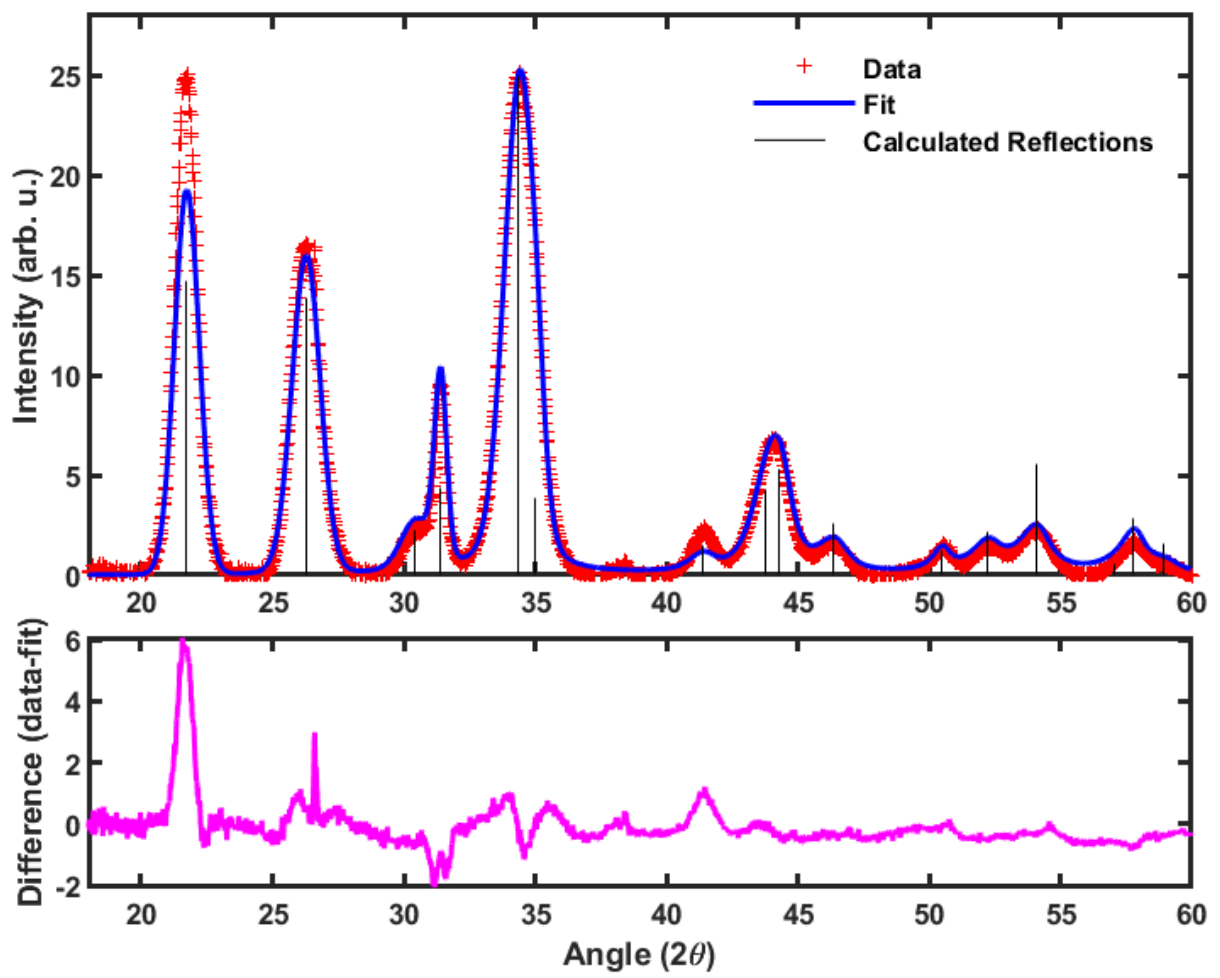

Figure S26. Data from a sample of nanorods ( $7 \mathrm{~nm}$ width, $50 \mathrm{~nm}$ length) synthesized by hot-injection under standard conditions. Refinement results: $a=6.778(7) \AA, c=5.695(6) \AA$; March-Dollase Parameter $=1.050(8) ; \mathrm{R}=17.0 \%$. 
Nanorod Sample, Example \#2

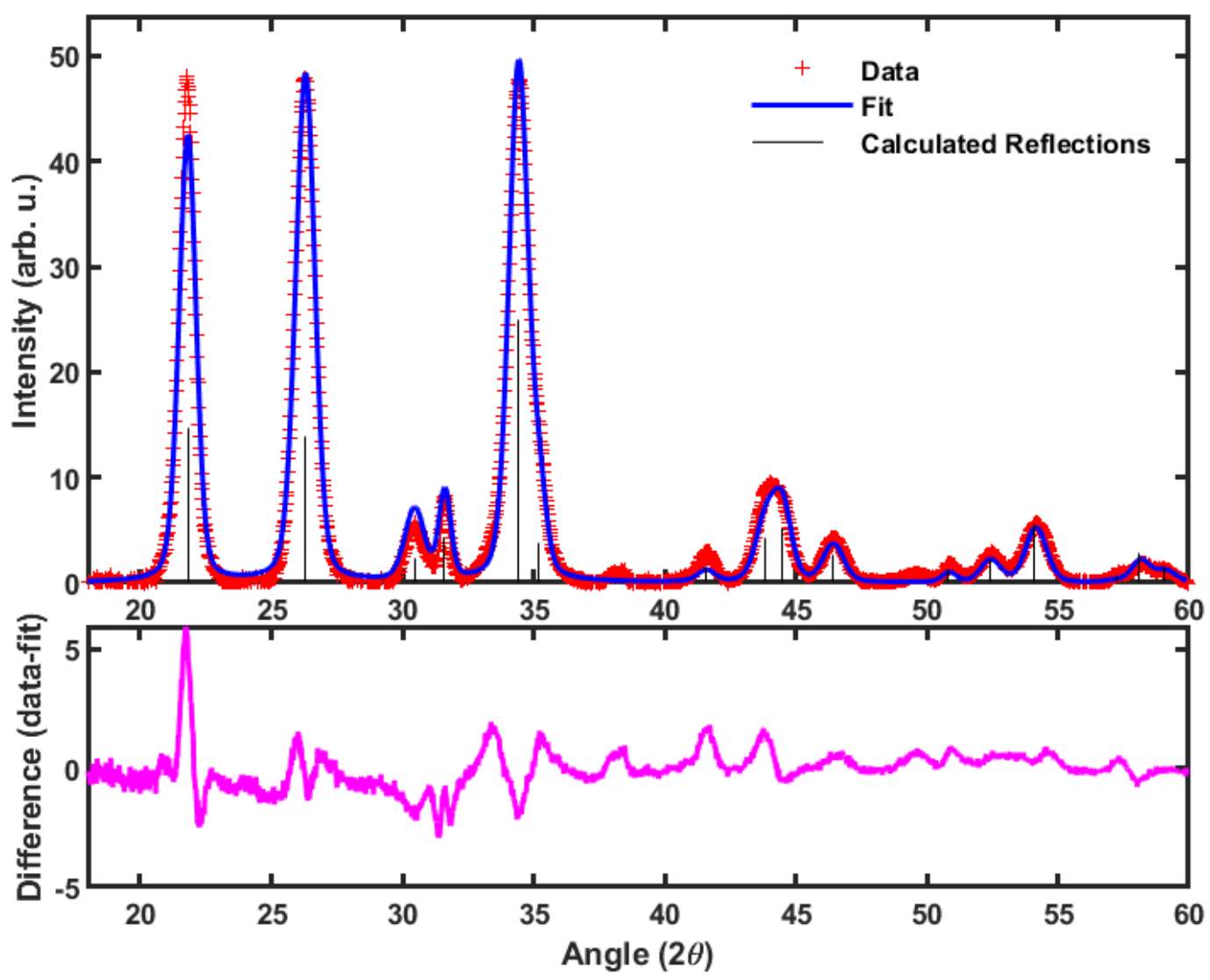

Figure S27. Data from a sample of nanorods ( $11 \mathrm{~nm}$ width, $28 \mathrm{~nm}$ length) synthesized by hot-injection under dilute conditions (13.6 mM). Refinement results: $a=6.772(4) \AA, c=5.656(4) \AA$; March-Dollase Parameter $=1.234(6) \mathrm{R}=17.4 \%$. 

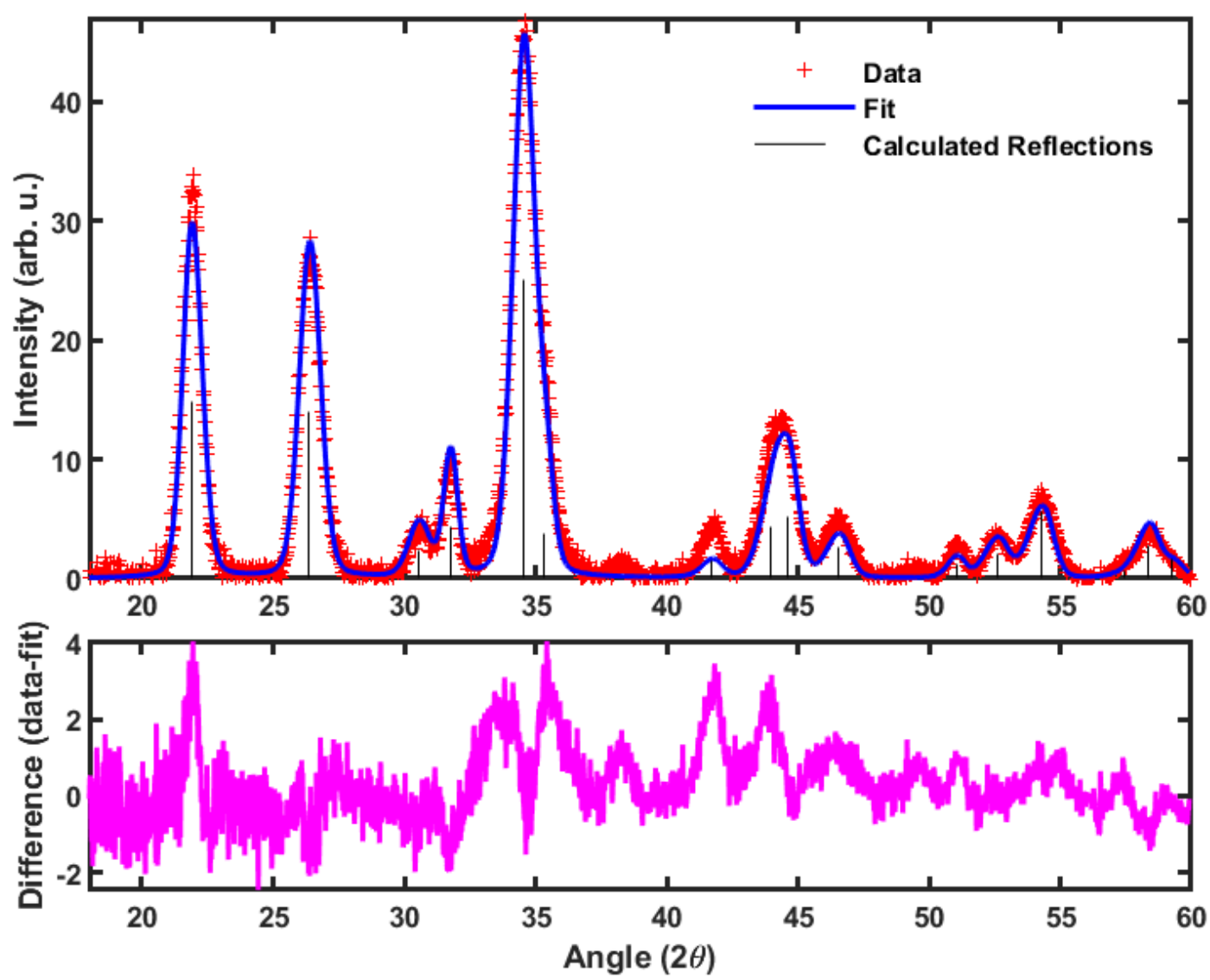

Figure S28. Data from a sample of nanorods ( $7 \mathrm{~nm}$ width, $12 \mathrm{~nm}$ length) synthesized by hot-injection under dilute conditions (16.3 mM). Refinement results: $a=6.752(4) \AA, c=5.633(4) \AA$; March-Dollase Parameter $=1.076(6) ; \mathrm{R}=21.2 \%$. 

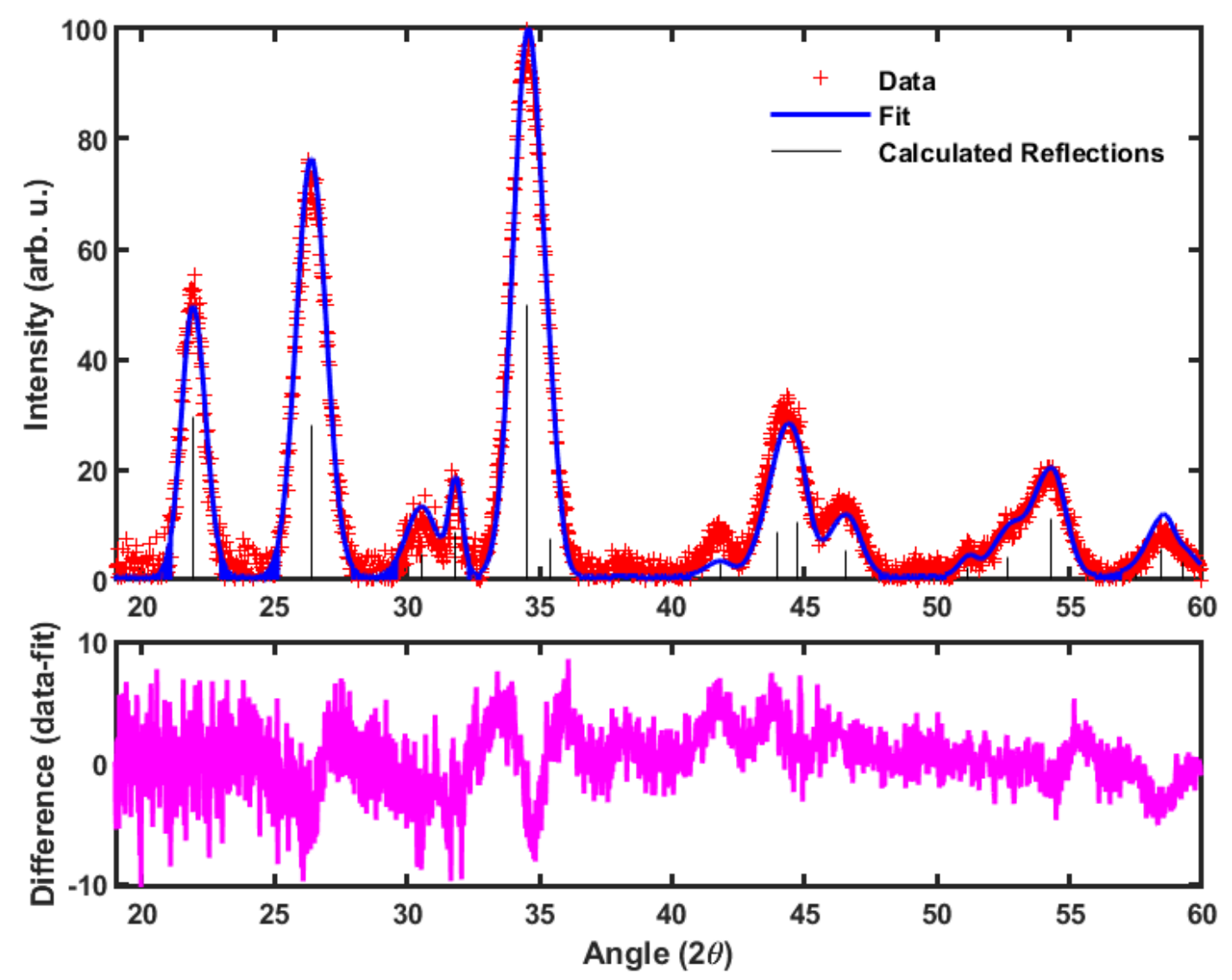

Figure S29. Data from a sample of nanorods ( $6.6 \mathrm{~nm}$ width, $38 \mathrm{~nm}$ length) synthesized by hot-injection under standard conditions except with a 2 hour reaction time. Refinement results: $a=6.753(6) \AA, c=$ $5.618(5)$ Å; March-Dollase Parameter $=1.319(8) ; R=30.4 \%$. 

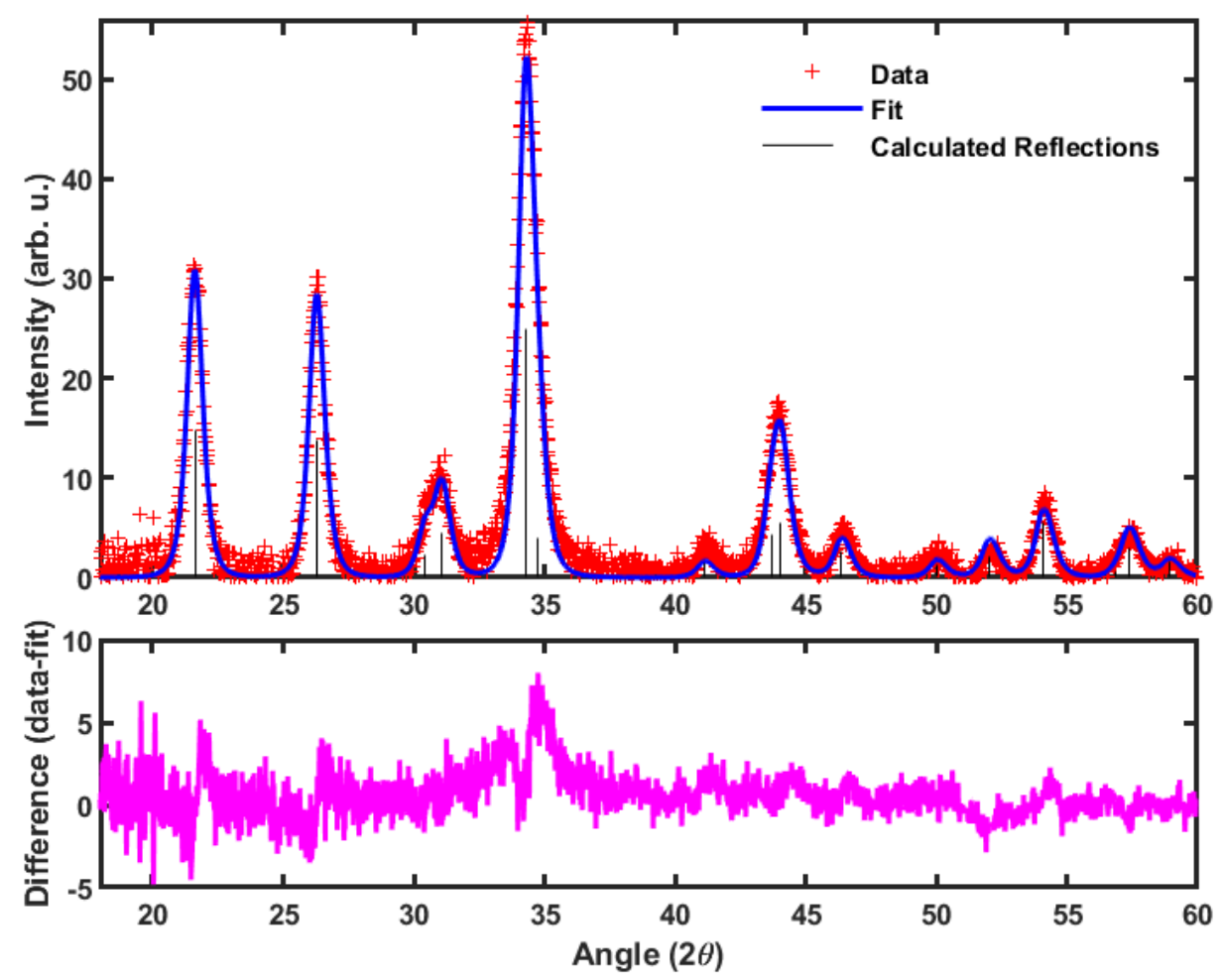

Figure S40. Data from a sample of nanoparticles $(12 \mathrm{~nm})$ synthesized under standard conditions. Refinement results: $a=6.776(4) \AA, c=5.750(4) \AA ̊$; March-Dollase Parameter $=0.94(1) ; R=36 \%$.

\section{Supplemental References.}

1. Vaartstra, B. A.; Huffman, J. C.; Streib, W. E.; Caulton, K. G., Syntheses and structures of a series of very low coordinate barium compounds: $\left.\mathrm{Ba}\left[\mathrm{N}\left(\mathrm{SiMe}_{3}\right)_{2}\right]_{2}(\mathrm{THF})_{2},\left\{\mathrm{Ba}\left[\mathrm{N}\left(\mathrm{SiMe}_{3}\right)_{2}\right)\right]_{2}(\mathrm{THF})\right\}_{2}$, and

$\left\{\mathrm{Ba}\left[\mathrm{N}\left(\mathrm{SiMe}_{3}\right)_{2}\right]_{2}\right\}_{2}$. Inorg. Chem. 1991, 30 (1), 121-125.

2. Plashnitsa, V. V.; Vietmeyer, F.; Petchsang, N.; Tongying, P.; Kosel, T. H.; Kuno, M., Synthetic strategy and structural and optical characterization of thin highly crystalline titanium disulfide nanosheets. J. Phys. Chem. Lett. 2012, 3, 1554-1558.

3. Zhu, Z.; Zou, Y.; Hu, W.; Li, Y.; Gu, Y.; Cao, B.; Guo, N.; Wang, L.; Song, J.; Zhang, S.; Gu, H.; Zeng, H., Near-infrared plasmonic 2D semimetals for applications in communication and biology. Adv.

Func. Mater. 2016, 26 (11), 1793-1802.

4. Clearfield, A., The synthesis and crystal structures of some alkaline earth titanium and zirconium sulfides. Acta Cryst. 1963, 16, 135-142.

5. Huster, J., Die Kristallstruktur von $\mathrm{BaTiS}_{3}$. Z. Naturforsch. B 1980, 35, 775.

6. Saeki, M.; Onoda, M.; Yajima, Y., Composite crystals BaTiS $(y=2.70-2.93)$. J. Solid State Chem. 1996, 121, 451-456. 